\title{
A Novel Memristor Chaotic System with a Hidden Attractor and Multistability and Its Implementation in a Circuit
}

\author{
Lili Huang, ${ }^{1}$ Yanling Wang, ${ }^{1}$ Yicheng Jiang, ${ }^{2}$ and Tengfei Lei ${ }^{1}{ }^{1}$ \\ ${ }^{1}$ Collaborative Innovation Center of Memristive Computing Application, Qilu Institute of Technology, Shandong, Jinan 250200, \\ China \\ ${ }^{2}$ School of Electronic Information, Nanjing University of Information Technology, Nanjing 210000, China
}

Correspondence should be addressed to Tengfei Lei; leitengfei2017@qlit.edu.cn

Received 30 April 2021; Accepted 26 May 2021; Published 2 June 2021

Academic Editor: Yi Qi

Copyright (c) 2021 Lili Huang et al. This is an open access article distributed under the Creative Commons Attribution License, which permits unrestricted use, distribution, and reproduction in any medium, provided the original work is properly cited.

\begin{abstract}
By introducing an ideal and active flux-controlled memristor and tangent function into an existing chaotic system, an interesting memristor-based self-replication chaotic system is proposed. The most striking feature is that this system has infinite line equilibria and exhibits the extreme multistability phenomenon of coexisting infinitely many attractors. In this paper, bifurcation diagrams and Lyapunov exponential spectrum are used to analyze in detail the influence of various parameter changes on the dynamic behavior of the system; it shows that the newly proposed chaotic system has the phenomenon of alternating chaos and limit cycle. Especially, transition behavior of the transient period with steady chaos can be also found for some initial conditions. Moreover, a hardware circuit is designed by PSpice and fabricated, and its experimental results effectively verify the truth of extreme multistability.
\end{abstract}

\section{Introduction}

In 1963, the first chaotic system was discovered by Lorenz. Since then, many scientists have constructed many new chaotic systems, such as Chen system, Lu system, and Jerk system $[1,2]$. Then, in 1971, Chua proposed the memristor, the fourth element after resistor [3, 4], capacitor, and inductor. People have studied chaotic systems based on memristor design. Compared with other classical chaotic systems [5], memristive nonlinear systems have more complex chaotic characteristics [6-10]. Some articles reported the phenomenon of memristive multistable state.

In addition to the sensitivity of the system to the parameters, it also depends on the initial value of the memristor. Due to the introduction of the ideal memristor [11-16], the dynamic system based on the memristor produces infinite equilibria, such as line equilibria and surface equilibria. These equilibria are related to the initial state variables of the memristor. These memristor models not only exhibit complex chaotic behaviors but also produce multistability phenomena $[17,18]$. As we all know, multistability is the coexistence behavior of two or more attractors under the same parameters and different initial conditions [19-21]. Bao et al. [22] introduced an ideal and active flux-controlled memristor into an existing hypogenetic chaotic jerk system, an interesting memristor-based chaotic system with a hypogenetic jerk equation, and proposed circuit forms. The most striking feature is that this system has four line equilibria and exhibits the extreme multistability phenomenon of coexisting infinitely many attractors. Jafari et al [23] propose a newly parameter estimation method on both an ordinary chaotic system and a chaotic system with extreme multistability. It proves the importance of that difference better by comparing the efficiency of the chaotic system. Recently, the introduction of trigonometric functions, such as the tangent function, sine function, hyperbolic tangent function [24, 25], and hyperbolic sine function, has made the system produce multiscroll attractors, multiwing attractors, infinite coexistence attractors, and attractor duplication phenomena [26, 27]. This nonlinear performance is caused by the periodicity of trigonometric functions $[28,29]$. This special feature has been reported in some chaotic article recently. However, there are relatively few studies on homogeneous multistability in the memristor chaotic systems of trigonometric functions [30-34]. 
Inspired by the abovementioned ideas, an interesting memristor-based chaotic system is constructed in this paper, which is achieved by introducing a tangent function $(\tan (z))$ and an ideal and active flux-controlled memristor with absolute value nonlinearity into an existing chaotic system boostable VB18 [31]. The newly proposed memristive system has infinite line equilibria and can exhibit the initialcondition-dependent extreme multistablity phenomenon of coexisting infinitely many attractors, which has seldom been reported in the academic literature.

The rest of this paper is organized as follows. In Section 2, a novel memristive system with infinite line equilibria is presented, upon which the stability for the infinite line equilibria are explored. In Section 3, parameter-dependent change is investigated by bifurcation diagrams and Lyapunov exponent spectra and coexisting intermittent chaos behavior revealed by phase portraits. In Section 4, initial-conditiondependent extreme multistability is investigated by coexisting bifurcation diagrams and Lyapunov exponent spectra, and coexisting infinitely many attractors' behavior and symmetric behavior are revealed by phase portraits. In Section 5, an implementation circuit is designed and PSpice circuit simulations are performed to verify the initial-condition-dependent dynamical behaviors of coexisting infinitely many attractors. In Section 6, the conclusions are summarized.

\section{Model Description}

According to the chaotic system VB18 reported in [31], a new memristive system can be easily constructed by the tangent function to substitute a $z$ status variable and by introducing utilizing memristor in the chaotic system with an existing nonlinear dynamical system boostable VB18.

A VB18 chaotic system is described as

$$
\left\{\begin{array}{l}
\dot{x}=a z+y^{2}-1, \\
\dot{y}=b y z \\
\dot{z}=-x-z .
\end{array}\right.
$$

The memductance function of the desired flux-controlled memristor is expressed as

$$
W(\phi)=-m+n|\phi|,
$$

where $m$ and $n$ are two memristor parameters with positive values. The abovementioned model (2) is used to describe an ideal and active flux-controlled memristor with an absolute value nonlinearity.

Through introducing the newly proposed memristor featured by (2) and trigonometric function $(\tan (z))$ into the chaotic system in [1], a new kind of memristor chaotic system is established, which can be mathematically modeled as

$$
\left\{\begin{array}{l}
\dot{x}=a \tan (z)+y^{2}-1 \\
\dot{y}=b y \tan (z) \\
\dot{z}=-x W(u)-\tan (z) \\
\dot{u}=x
\end{array}\right.
$$

where $x, y, z$, and $u$ are the state variables of system (3), $a$, $b$, and $c$ are the control parameters of system (3), and
$W(u)=-m+n|u|$ is the normalized memductance function. $u$ in formula (3) is $\varphi$ in formula (2).

The voltage-current curves of the memristor with different values of the frequency $f$ are plotted in Figure 1. It can be seen that the pinched hysteresis loop gradually shrinks with $f$ increasing from 1.07 to 2.07 , then to 3.07 in Figure 1(a), and keeps pinched at the original point with the origin, which is a typical 8-like hysteresis loop. It can be seen that $W(u)$ stands for the memductance related to the magnetic flux $u$ in Figure 1(b) implying that system (2) satisfies the definition of the memristor.

When $a=5.8, b=7.9, m=0.02$, and $n=0.06$, the initial conditions $\left(x_{0}, y_{0}, z_{0}, u_{0}\right)$ are assigned as $(1,1,0,5)$. Matlab numerical simulations are performed and several useful results are obtained, as shown in Figure 2, from which the chaotic behavior characterized by a strange attractor is revealed. System (3) displays two phase portraits in two different planes with a single-scroll attractor and doublevortex attractor. Correspondingly, the finite-time Lyapunov exponents are calculated by Wolf s method [33] as $L 1=0.041139, L 2=-0.002245, L 3=-0.733286, L 4=-1.475$ 227 , and Kaplan-York dimension DKY $=3+(L 1+L 2+L 3) /$ $L 4=3.4707$. Consequently, the numerical results in Figure 2 indicate that system (3) is a chaotic system.

\section{Basic Dynamical Analysis}

3.1. Stability Analysis of the Equilibrium Point. When $a=5.8$, $b=7.9, m=0.02$, and $n=0.06, x, y, z$, and $w$ are state variables of system (3). The equilibrium points of system (3) can be easily calculated by setting the left-hand side to zero, which are not associated with the memductance function.

$$
\left\{\begin{array}{l}
a \tan (z)+y^{2}-1=0 \\
b y \tan (z)=0 \\
-x(-m+n|u|)-\tan (z)=0 \\
x=0
\end{array}\right.
$$

According to the fourth dimension $x=0$ of equation (4), it can be that the third dimension is $\tan (z)=0$, and the solution $z(z=k \pi)(k \in N)$; then, $y^{2}-1=0$ in the first dimension can get $y= \pm 1$, and then, system (3) has an infinite equilibrium point $(0, \pm 1, k \pi, u),(k \in N)$, where $k$ is any real constant.

The Jacobian matrix of system (3) at the equilibrium point $E=(1,1,0, u)$ is yielded as

$$
J e c=\left[\begin{array}{cccc}
0 & 2 y & a(\sec (z))^{2} & 0 \\
0 & b \tan (z) & b y(\sec (z))^{2} & 0 \\
-W(u) & 0 & -(\sec (z))^{2} & -x n \operatorname{sign}(u) \\
1 & 0 & 0 & 0
\end{array}\right],
$$

where $W(u)=-m+n|u|$. In consideration of $z=0, u=h$, the characteristic polynomial equation is derived as

$$
\lambda\left(a_{0} \lambda^{3}+a_{1} \lambda^{2}+a_{2} \lambda^{1}+a_{3}\right)=0
$$

where $a_{1}=a_{0}=1, \quad a_{2}=0.348|h|-0.116$, and $a_{3}=0.948$ $|h|-0.316$, and the abovementioned characteristic 


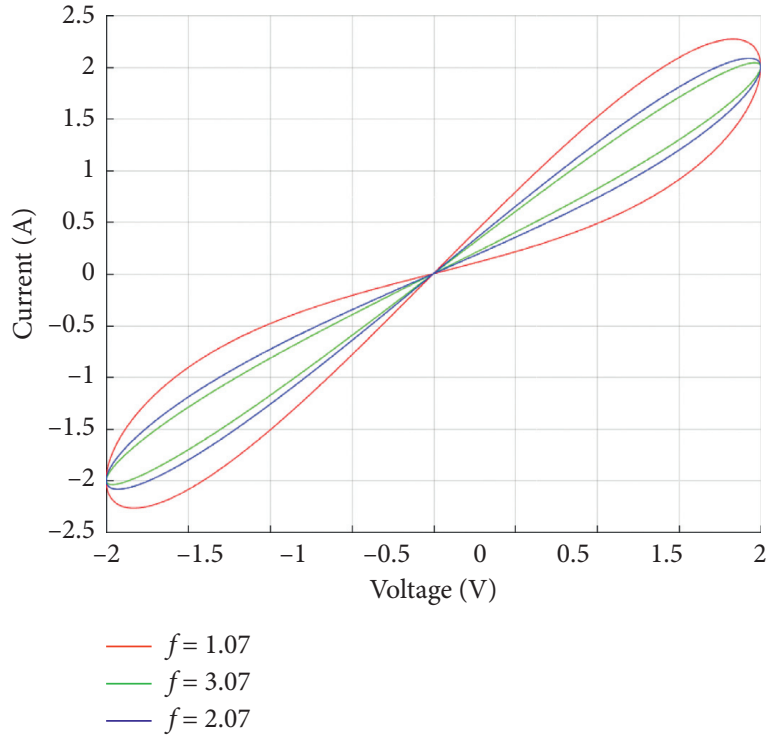

(a)

Figure 1: The memductance and pinched hysteresis loop. (a) The pinched hysteresis loop; (b) the memductance loop.

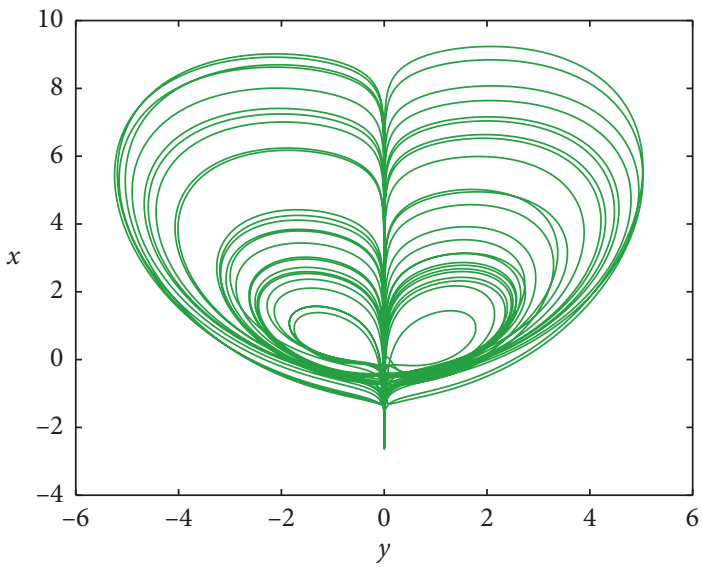

(a)

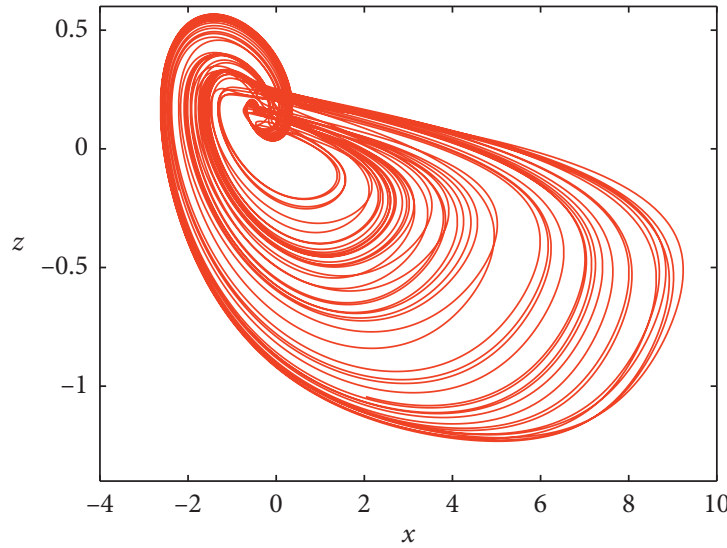

(c)

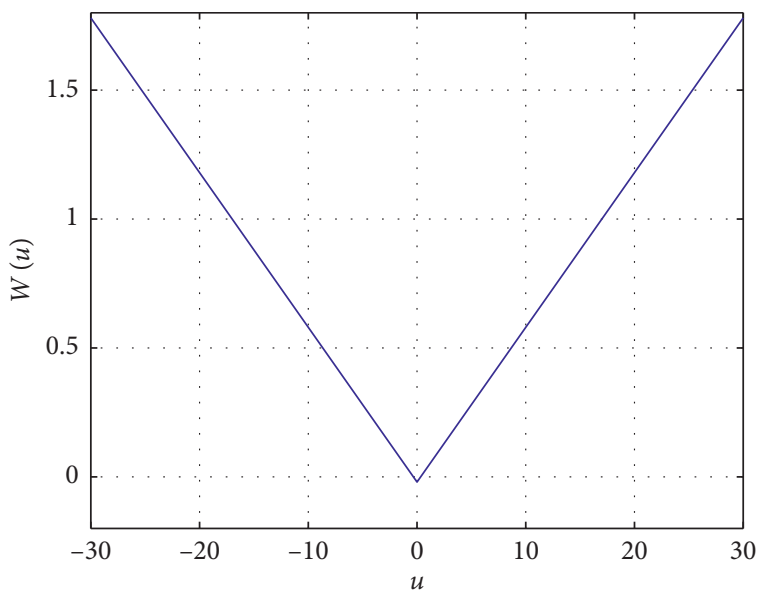

(b)

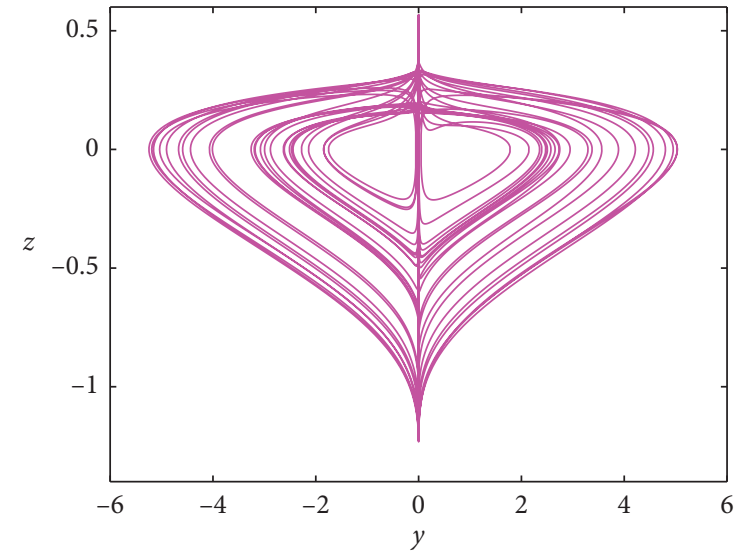

(b)

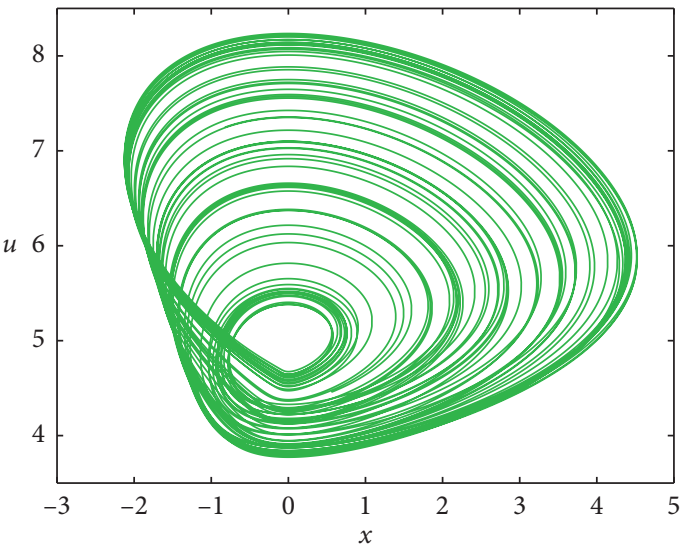

(d)

FIGURE 2: Chaotic behavior characterized by an symmetric chaotic attractor of system (2) with $a=5.8$ and $b=7.9$ under initial condition $[1,1,0,5]$ : (a) phase portrait in the $x$ - $y$ plane, (b) phase portrait in the $y$ - $z$ plane, (c) phase portrait in the $z$ - $x$ plane, and (d) phase portrait in the $x$-u plane. 
polynomial implies that Jacobian matrix (5) has three nonzero roots and one zero root. For these roots, Routh-Hurwitz conditions are given as

$$
H_{k}=\left|\begin{array}{ccc}
a_{1} & a_{3} & a_{5} \\
a_{0} & a_{2} & a_{4} \\
0 & a_{1} & a_{3}
\end{array}\right|>0,
$$

where $k=1,2,3$, and

$$
\begin{aligned}
H_{1} & =a_{1}>0, \\
H_{2} & =a_{1} a_{2}-a_{3}>0, \\
H_{3} & =a_{3}\left(a_{1} a_{2}-a_{3}\right)>0, \\
\text { i.e., } 0.348|h| & -0.116+0.316-0.948|h|<0 .
\end{aligned}
$$

If the conditions in (8) are satisfied, i.e., $-(1 / 3)<h<$ $(1 / 3), E$ is stable. Obviously, the eigenvalues of (6) are relevant to the algebraic symbols $u$. When $h \geq(1 / 3)$ or $h \leq-(1 / 3)$, system (3) has one positive real root and two complex conjugate roots with negative real parts. The results imply that the infinite line equilibiria of system (3) only consist of unstable saddle points and unstable saddle-foci.

3.2. Bifurcation Analysis. The dynamic properties of the system will be changed by the changing parameters, which will make the system show the phenomena of chaotic periodic divergence.

For system (3), the parameters $a$ and $b$ effectively determine the dynamic behavior, as shown in Figure 3. When the initial value $[1,1,0,5]$ is fixed, the parameter $b=7.9$. When the parameter $a$ varies in $[2,11]$, chaos and periodic oscillation appear alternately as shown in Figure 3. The largest finite-time Lyapunov exponents are calculated and plotted with varying parameter $a$, as shown in Figure 3. As we can be seen from Figure 3, when $a$ is in the interval $[6.23,6.355]$ and $[4.256,4.455]$, the largest finite-time Lyapunov exponents are equal to 0 , and the system produces cycle- 4 attractors. When $a$ is in the interval $[4.85,4.95]$ and $[6.25,7.25]$, the system produces cycle-2 attractors. When $a$ is in the interval $[7.35,11]$, the system produces cycle- 1 attractors. The change of parameter $a$ makes system (3) show inverted period bifurcation behavior. When $a>7.2$, the antiperiod bifurcation of the system has chaos and reaches a steady state. Then, the same initial value $[1,1,0,5]$ is fixed, when the parameter $a=5.8$, and the parameter $b$ changes in $[4,13]$; the Lyapunov exponent spectrum and bifurcation diagram of system (3) are shown in Figures 4(a) and 4(b). The change of parameter $b$ makes system (3) show period-doubling bifurcation behavior. It can be seen from Figure 4 that when the value of $b$ is in the interval $[7.566,8.26]$ and $[11.533,12.155]$ and $[12.566,13.215]$, the system is in a chaotic state. Obviously, when parameter $b$ is set to different values, the system shows periodic, chaotic, single scroll, double scroll, and other dynamic behaviors as shown in Figure 5.

\section{Multistable State Analysis}

When the same parameter takes different initial values, two or more attractors are called coexistent attractors or multiple attractors, which is called the multistable state. The fourdimensional initial value of system (3) has different attractors when it takes different values. It can generate various types of coexisting attractors.

4.1. Dynamics with Respect to Memristor Initial Condition $\left(u_{0}\right)$. In most memristor systems, the value of a memristor has some relations with the initial condition, so we have different oscillating dynamics depending on whether the initial data of the internal variable causes different oscillations. This phenomenon is widely studied and is known as extreme polystability. In system (3), the control parameters are set as $a=5.8, b=7.9, m=0.02$, and $n=0.06$, the initial conditions are assigned $x(0)=0, y(0)=1$, and $z(0)=0$, and the memristor initial condition $u(0)$ is taken as the bifurcation parameter. When the memristor initial condition $u_{0}$ varies in $(-30,30)$, system (3) has a different stable state, in which the Lyapunov exponent and bifurcation evolution are shown in Figures 6(a) and 6(b) and 7(a) and 7(b). When the memristor initial condition $u(0)$ is gradually changed, system (3) shows multiple period-doubling bifurcations and the chaotic state intermittent existence phenomenon. Consequently, the results of Figures 6 and 7 demonstrate that, under different memristor initial conditions, there are completely different dynamical behaviors in system (3), leading to the coexisting phenomenon of many attractors. As can be seen from Figure 6(a), with the change of initial value $u_{0}$, system (3) shows extremely complex dynamic behavior, including single period, double period, chaotic behavior, and period doubling. In particular, it can be found that the dynamic behavior of system (3) is extremely sensitive to the disturbance from small changes in initial conditions, and the initial value $u_{0}$ has a completely different dynamic behavior in the region around parameter 0 . It is remarked that when the initial conditions of $x(0), y(0)$, and $z(0)$ are assigned to different values as shown in Figure 7, system (3) has different bifurcation behaviors as the memristor initial condition $u(0)$ is varied, which further indicates that there exists coexisting infinitely many attractors' behavior in system (3).

To better represent extreme multisteadiness, the typical phase portraits of attractors under different $u_{0}$ are shown in Figure 8 . When the initial value $u_{0}$ is different, the state of system (3) is shown in Table 1.

\subsection{Dynamics with Respect to Memristor Initial Condition} $\left(Y_{0}\right)$. The control parameters of system (3) are kept unchanged, the initial conditions are assigned as ( $\left.\begin{array}{llll}1 & Y_{0} & 0\end{array}\right)$, and the initial condition $Y_{0}$ is taken as the bifurcation parameter. When $Y_{0}$ is varied in the region $[-10,10]$, the bifurcation diagram of the state variable $Y_{0}$ and its Lyapunov exponent spectra are plotted in Figures 9(a) and 9(b). When the initial condition $Y_{0}$ is increased from 0 , system (3) goes from the normal chaotic state into the quasiperiodic state, breaks into the weak chaotic state, and then, turns into the normal chaotic state. 


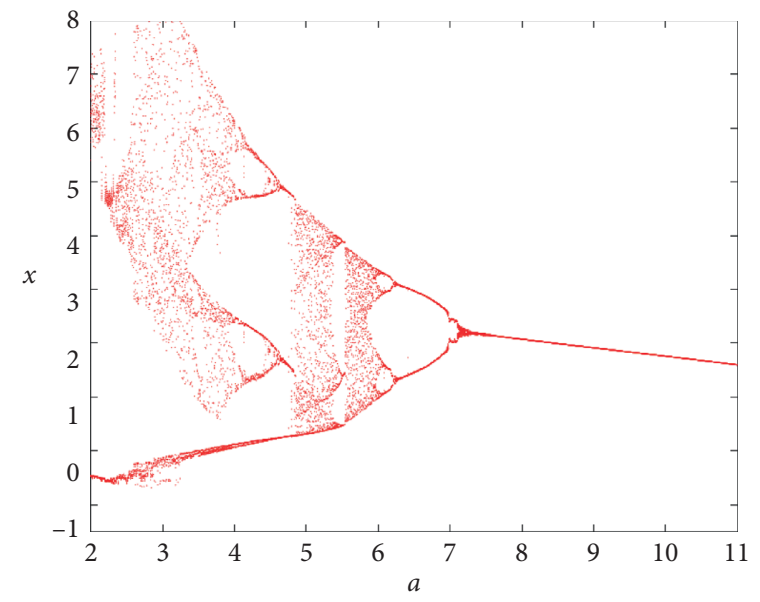

(a)

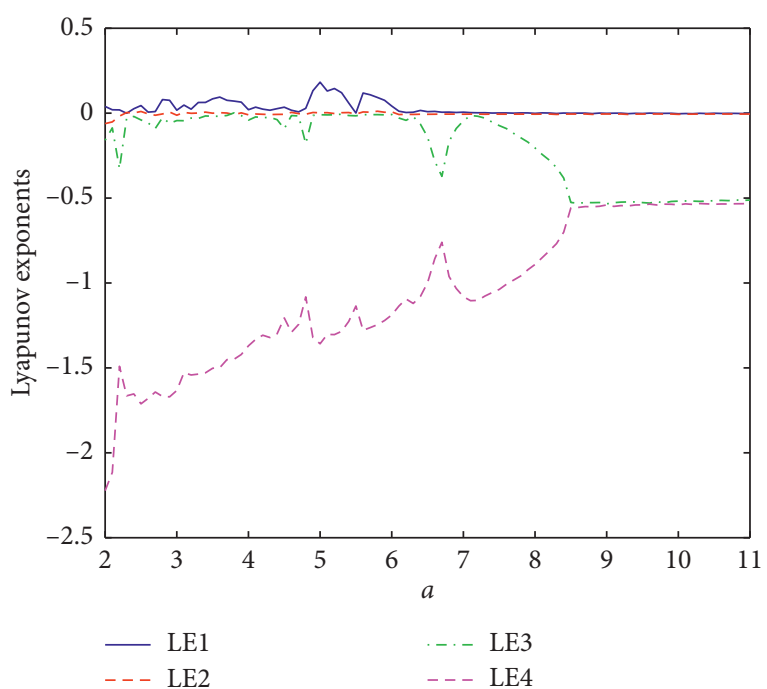

(b)

FigURE 3: Lyapunov exponents and bifurcation diagram of system (3) with $b=7.9$, when $a$ varies in $[2,11]$. (a) Bifurcation diagram; (b) Lyapunov exponents.

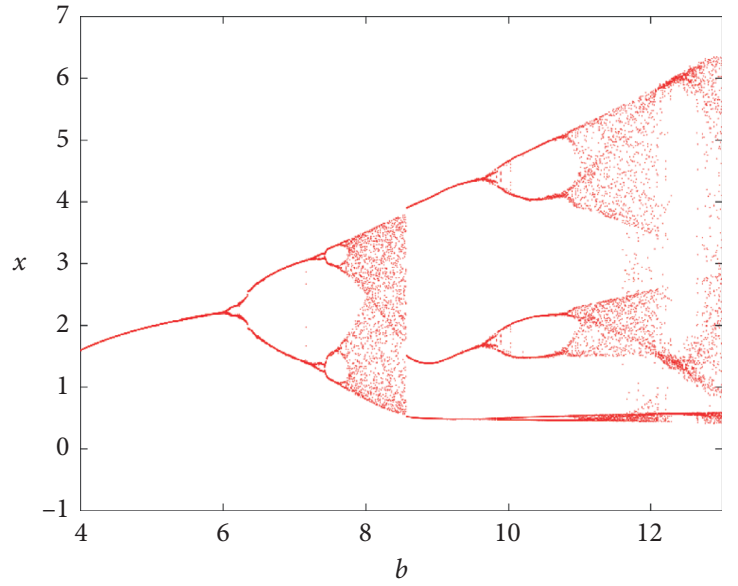

(a)

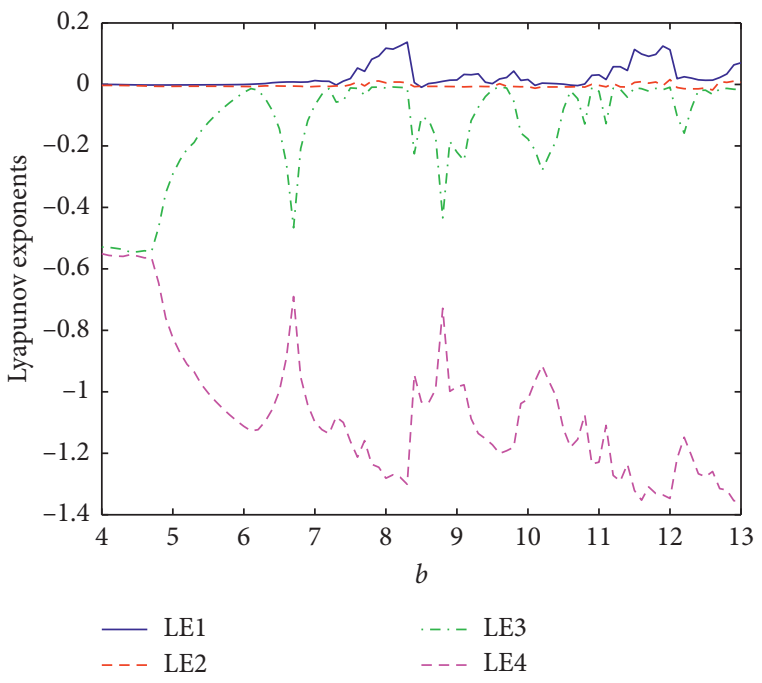

(b)

FiguRe 4: Lyapunov exponents and bifurcation diagram of system (3) with $a=5.8$, when $b$ varies in $[4,13]$. (a) Bifurcation diagram; (b) Lyapunov exponents.

Near $Y_{0}=1.85$, system (3) degrades into the periodic state via chaos, but near $Y_{0}=2.09$, system (3) abruptly changes into the chaotic state with periodic stats bifurcation. When $Y_{0}$ is further increased, system (3) jumps into the periodic state again at $Y_{0}=2.5$. In the parameter region $[1.85,2.09]$ of $Y_{0}$, system (3) mainly operates in the periodic state. In the parameter region $[2.13,8.45]$ of $Y_{0}$, system (3) mainly operates in the exhibits weak chaotic behaviors but exhibits weak chaotic behaviors near $Y_{0}=2.24,3.22$. Therefore, system (3) displays periodic state alternate with chaos. An example is taken to analyze the influence of the change of the initial value of $Y_{0}$ on the system. It can be seen that the initial value $Y_{0}$ is positive and negative, and system (3) has symmetry. When fixing $a=5.8$ and $b=7.9$ and initial value $Y_{0}$ changes in the interval $[-10,10]$, the phase diagram of the system is symmetrically distributed in the $x-y$ plane, as shown in Figure 10. When the value of $Y_{0}$ is 1.85 , the system is in the cycle- 3 stats, when the value of $Y_{0}$ is 2.215 , the system is in the cycle- 4 stats, and when the value of $Y_{0}$ is 3.214, the system is in the cycle- 6 stats. When the value of $Y_{0}$ is 1 , the system is in a chaotic state. Thus, the results of Figure 10 imply that there is coexisting many attractors' behavior in system (3).

4.3. Dynamics with Respect to Memristor Initial Condition $\left(z_{0}\right)$. Furthermore, for the periodic tangent function, all the infinite countless attractors can be self-reproduced in the 


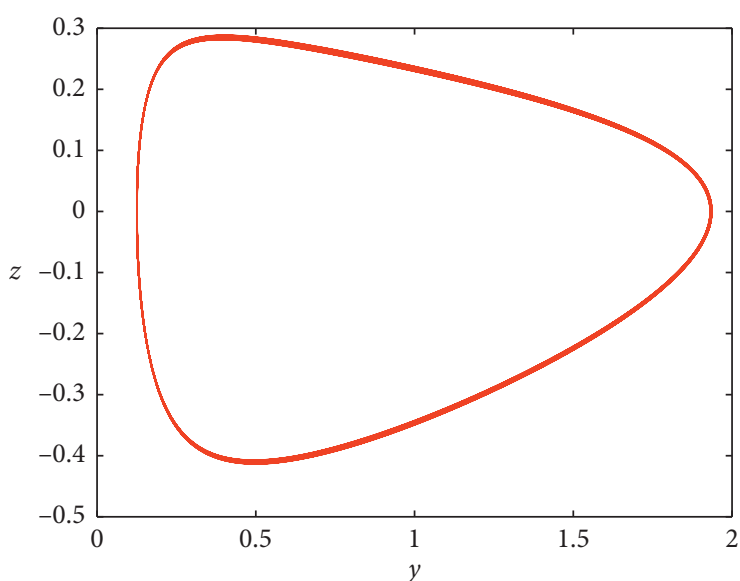

(a)

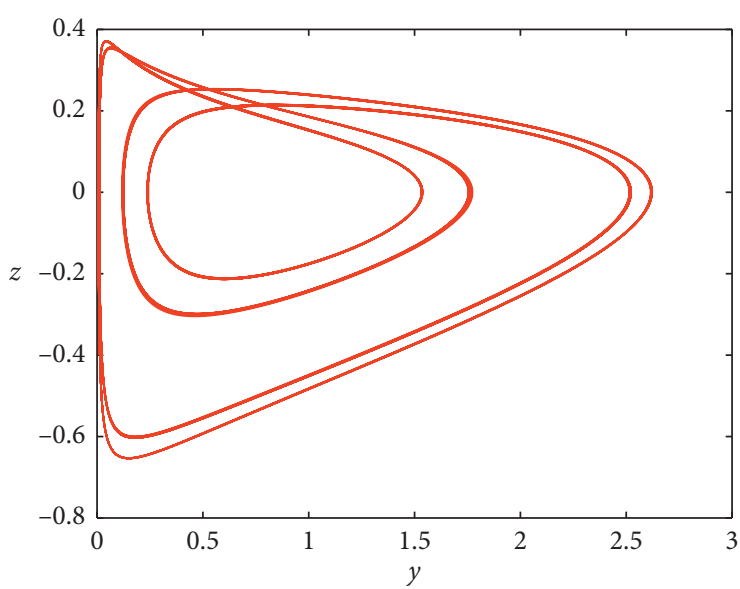

(c)

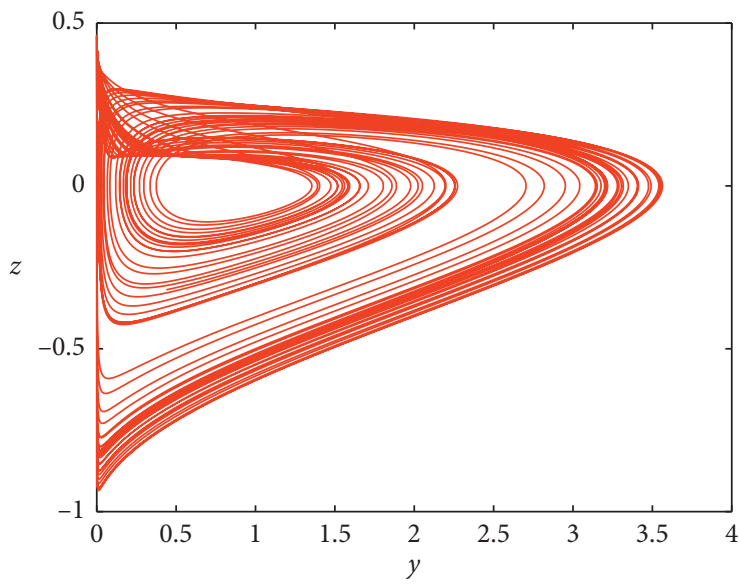

(e)

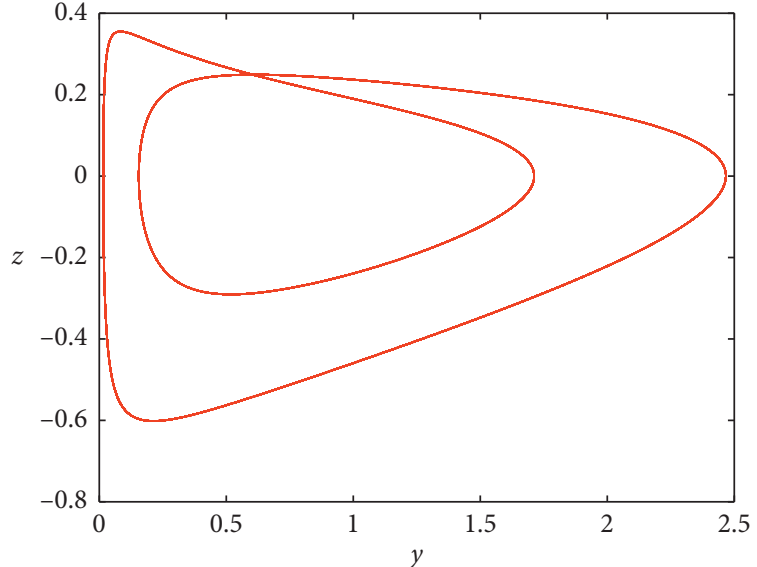

(b)

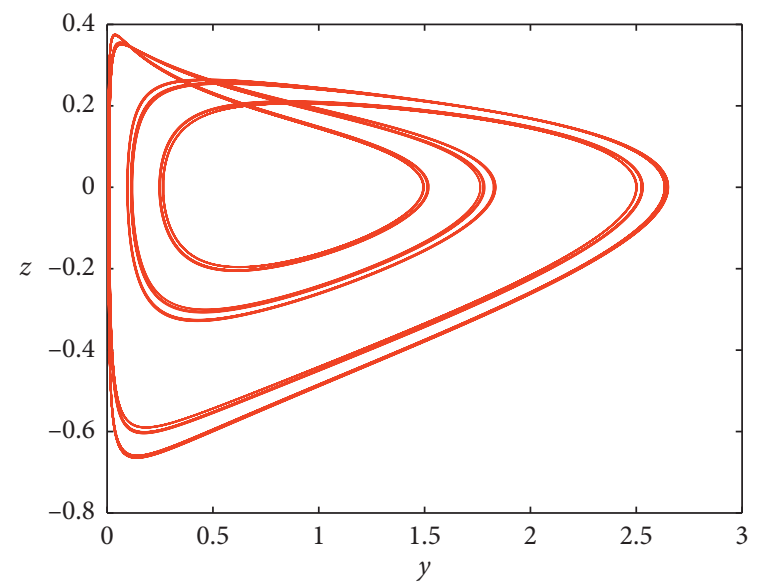

(d)

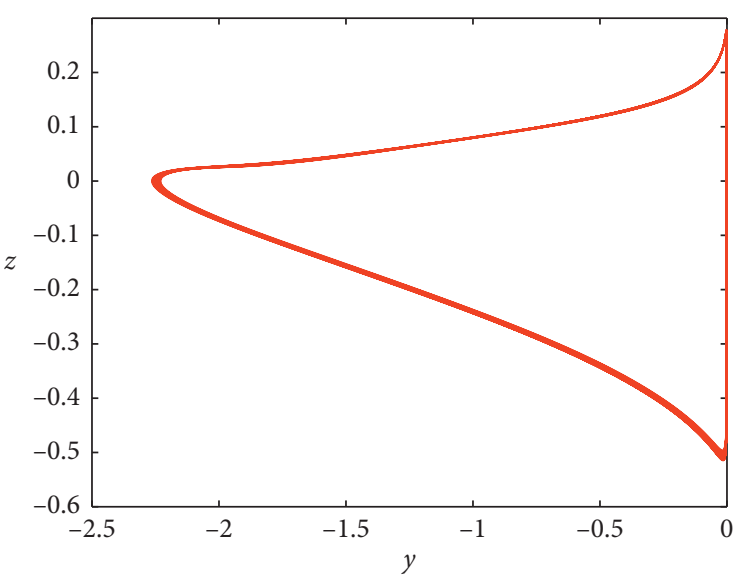

(f)

FIgURE 5: Continued. 


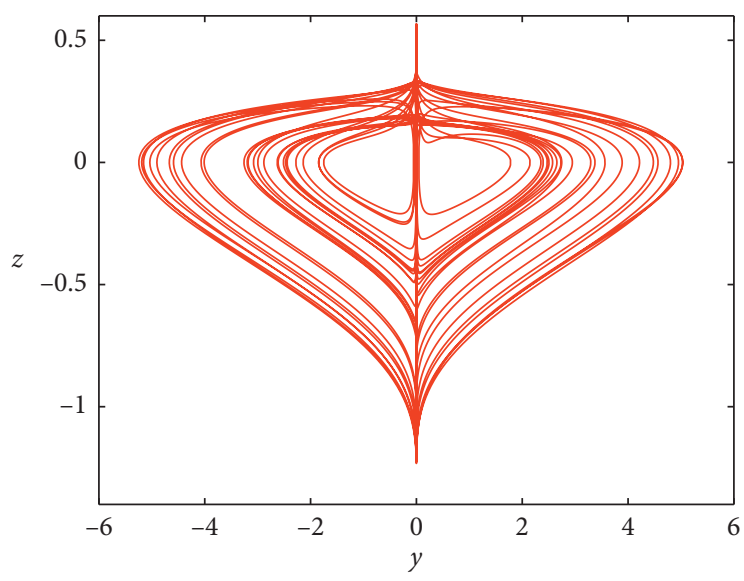

(g)

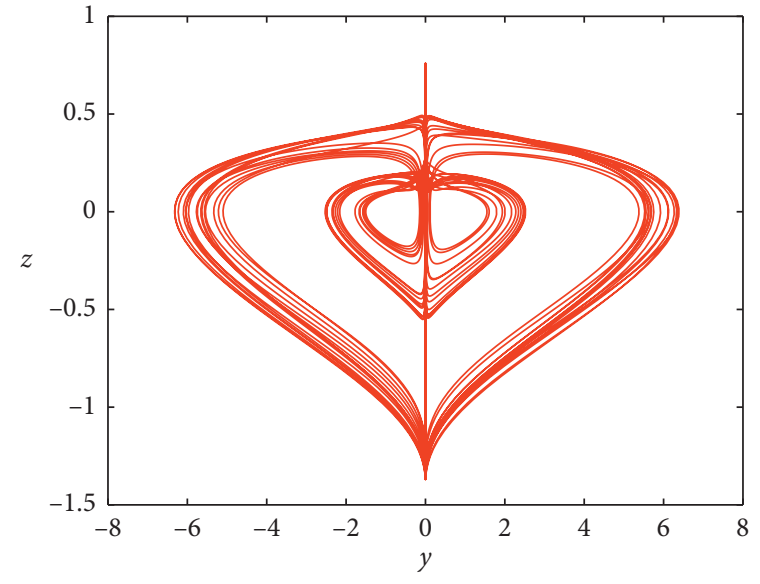

(h)

FIGURE 5: Phase portraits of attractors of system (3) with $a=5.8$ and IC $=(1,1,0,5)$ on the $z-y$ plane: (a) $b=5$, (b) $b=6.5$, (c) $b=7$, (d) $b=7.1$, (e) $b=10$, (f) $b=12.9833$, (g) $b=15$, and (h) $b=18$.

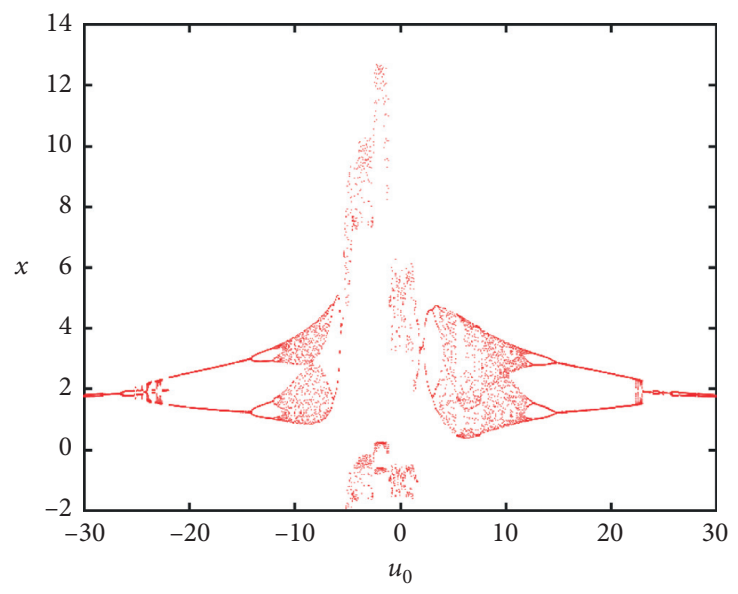

(a)

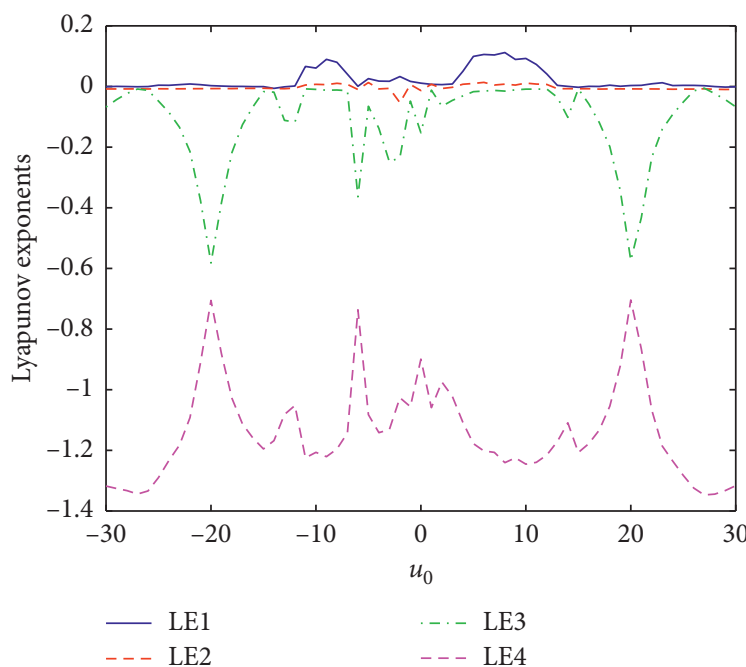

(b)

Figure 6: Dynamics with respect to $u(0)$, where $a=5.8$ and $b=7.9$ under the initial condition $\left[1,1,0, u_{0}\right]$. (a) Bifurcation diagram of the state variable $u\left({ }_{0}\right)$; (b) Lyapunov exponent spectra.

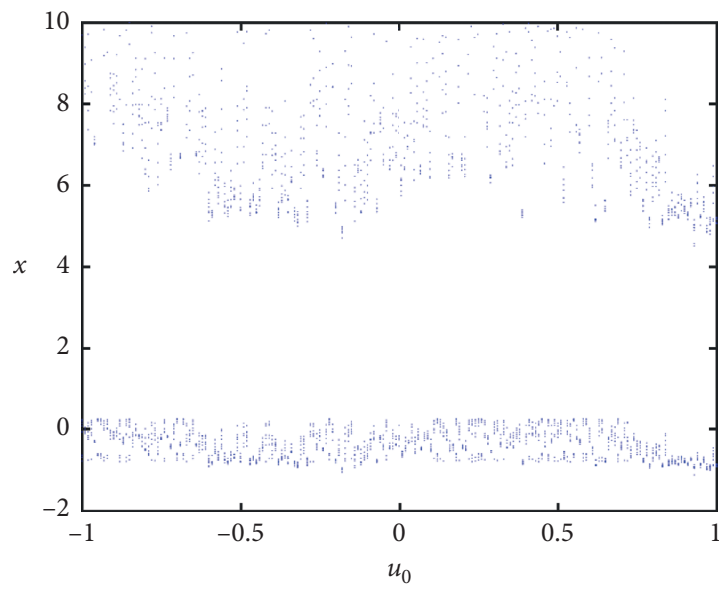

(a)

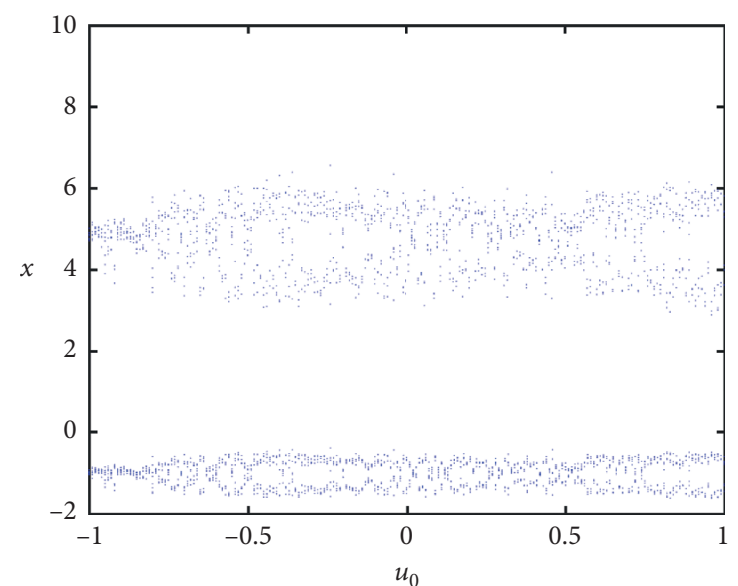

(b)

FIgURE 7: Bifurcation diagram of the state variable $u_{0} \in(-1,1)$. (a) The initial condition $\left(101-\right.$ pi $\left.u_{0}\right)$; (b) the initial condition $\left(110 u_{0}\right)$. 


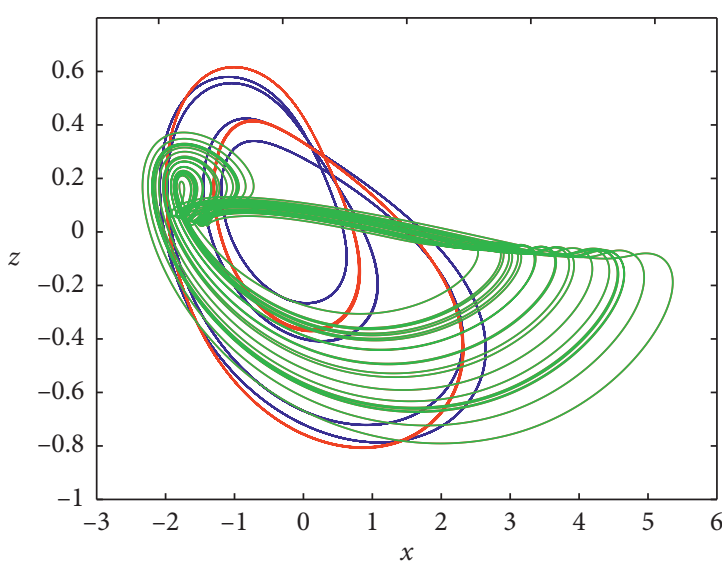

(a)

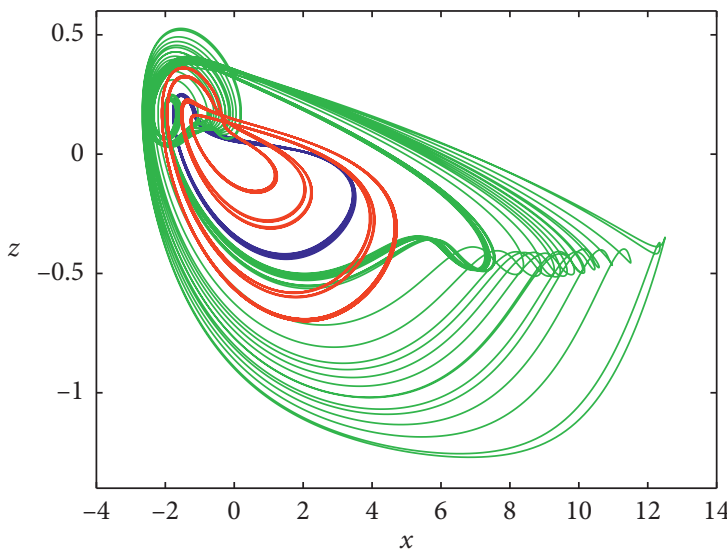

(c)

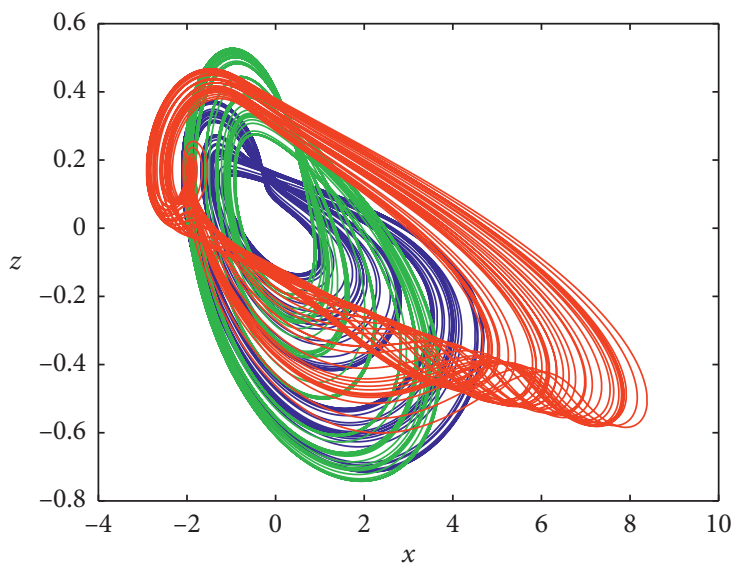

(e)

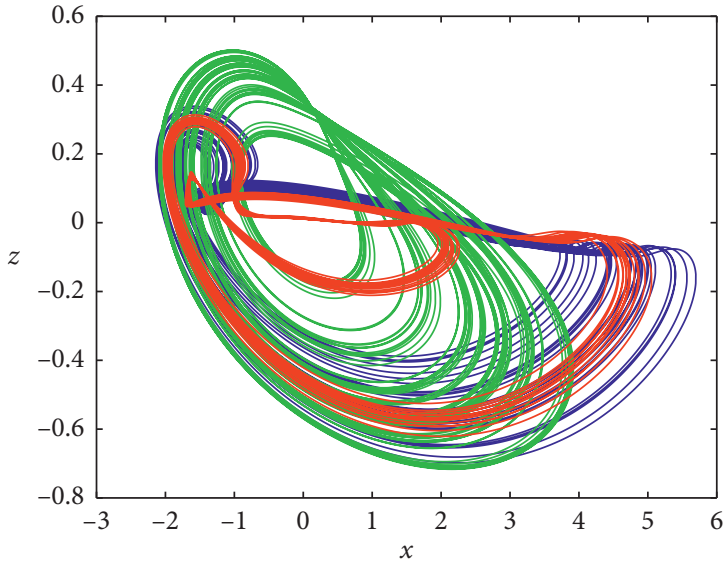

(b)

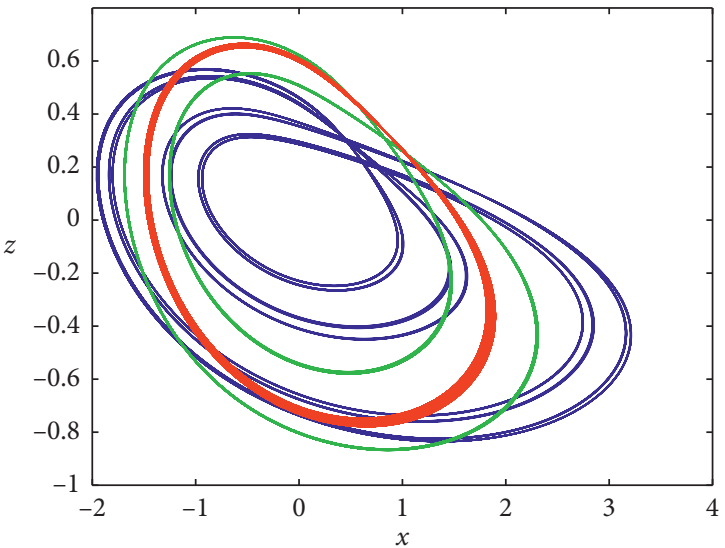

(d)

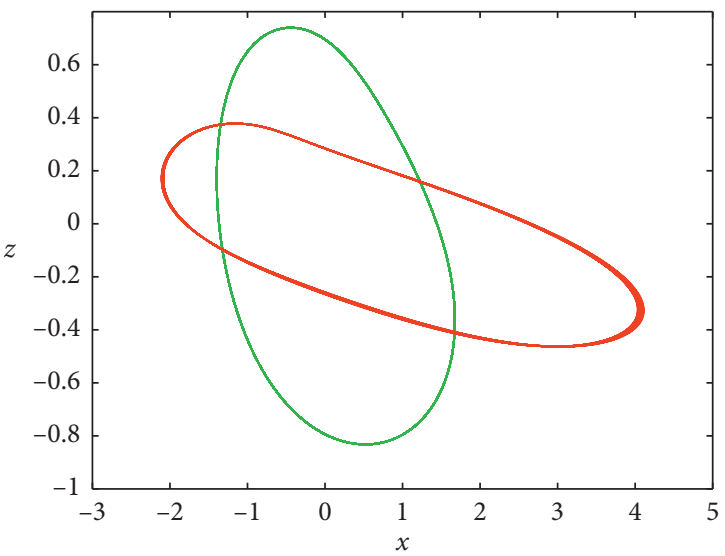

(f)

FIGURE 8: Coexisting attractor of system (2) with $a=5.8$ and $b=7.9$ under the initial condition IC1 [1, $\left.1,0, u_{0}\right]$. (a) $u_{0}=0.1$ (green), $u_{0}=-12.5$ (blue), and $u_{0}=-15$ (red); (b) $u_{0}=1.42$ (red), $u_{0}=-1$ (blue), and $u_{0}=-9$ (green); (c) $u_{0}=-2$ (green), $u_{0}=3.69$ (red), and $u_{0}=12.35$ (blue); (d) $u_{0}=25$ (red), $u_{0}=3.69$ (red), and $u_{0}=22.5$ (blue); (e) $u_{0}=-5$ (red), $u_{0}=-10$ (green), and $u_{0}=4$ (blue); and (f) $u_{0}=3.5$ (blue) and $u_{0}=-5.5$ (red).

dimension of $z_{0}$. System (3) parameters are chosen as $a=5.8$ and $b=7.9$, the value of $z_{0}$ is representatively $0, \pm \mathrm{pi}$, and $\pm 2 * \mathrm{pi}$, and five attractors appear in different positions, as shown in Figure 11. Thus, the results of Figure 11 imply that there is coexisting infinitely many attractors' behavior in system (3).
The bifurcation diagram and Lyapunov exponent spectrum under the change of the initial value $z_{0}$ are shown in Figure 12; it can be seen that when the initial value $z_{0}$ takes different values, system (3) changes from single-period, double-period, three-period and four-period, quasiperiodic, and chaotic attractors; here, four typical attractors including 
TABLE 1: Attractors in system (2) with $a=5.8$ and $b=7.9$ under initial conditions of $\left[1,1,0, u_{0}\right]$.

\begin{tabular}{lccc}
\hline Initial condition $\left(u_{0}\right)$ & Colour & Attractor & Figure number \\
\hline$u_{0}=0.1,-9,-2,-10$ & $\mathrm{~g}$ & Chaotic & $(a)-(c),(e)$ \\
$u_{0}=-12.5$ & $\mathrm{~b}$ & Cycle- & $(a)$ \\
$u_{0}=-15$ & $\mathrm{r}$ & Cycle-2 & $(a)$ \\
$u_{0}=1.42,-3.68$ & $\mathrm{r}$ & Quasiperiodicity & $(b)-(c)$ \\
$u_{0}=-1$ & $\mathrm{~b}$ & Chaotic & $(b)$ \\
$u_{0}=2.25$ & $\mathrm{~b}$ & Cycle-1 & $(\mathrm{c})$ \\
$u_{0}=22.5$ & $\mathrm{~g}$ & Cycle-2 & $(d)$ \\
$u_{0}=12.35$ & $\mathrm{~b}$ & Quasiperiodicity & $(d)$ \\
$u_{0}=25$ & $\mathrm{r}$ & Cycle-1 & $(d)$ \\
$u_{0}=-5$ & $\mathrm{r}$ & Chaotic & $(e)$ \\
$u_{0}=4$ & $\mathrm{~b}$ & Chaotic & $(e)$ \\
$u_{0}=35,-5.5$ & $\mathrm{~b}, \mathrm{r}$ & Cycle-1 & $(f)$ \\
\hline
\end{tabular}

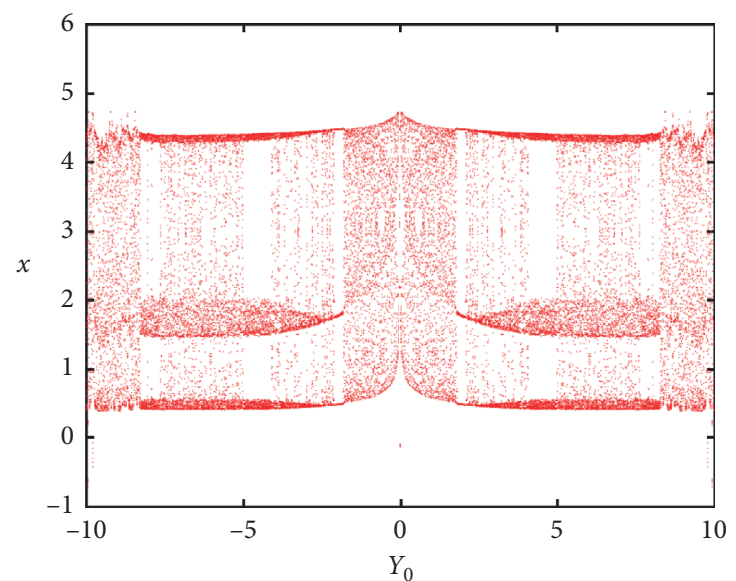

(a)

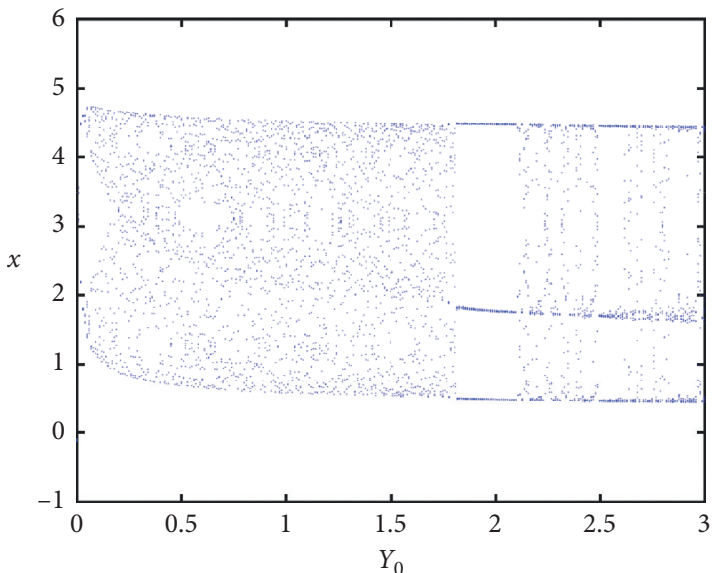

(c)

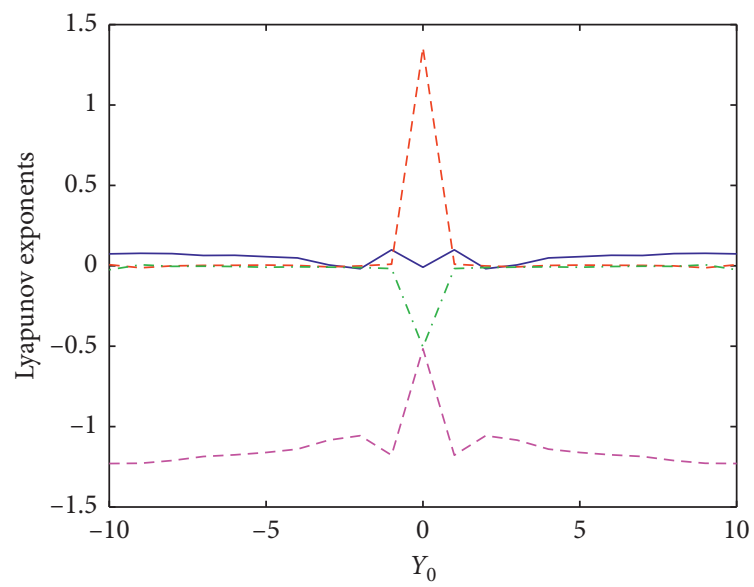

- LE1
--- LE2

- - - LE3

- - LE4

(b)

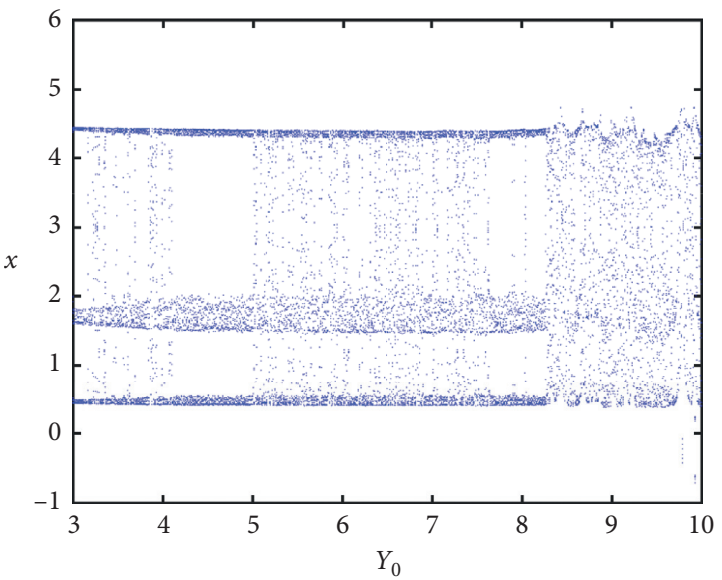

(d)

FiguRe 9: Lyapunov exponents and bifurcation diagram of system (2) with $a=5.8$ and $b=7.9$ under the initial condition [1, $\left.Y_{0}, 0,5\right]$. (a) Bifurcation diagram with $Y_{0} \in(0,10)$; (b) Lyapunov exponents; (c) bifurcation diagram with $Y_{0} \in(0,3)$; and (d) bifurcation diagram with $Y_{0} \in(3,10)$.

chaos and limit cycles are reproduced in phase space for clear demonstration. Simultaneously, the bifurcation diagram and the Lyapunov exponential spectrum are consistent. When $z_{0}$ is in the interval $[-0.55,0.8]$, the system is in a state of chaos and periodic state. Typical phase portraits of attractors of system (3) under different parameters are shown in Figure 13. When the value of $z_{0}$ is -0.55 , the system is in the cycle- 1 state, when the value of $z_{0}$ is -0.49 , the 


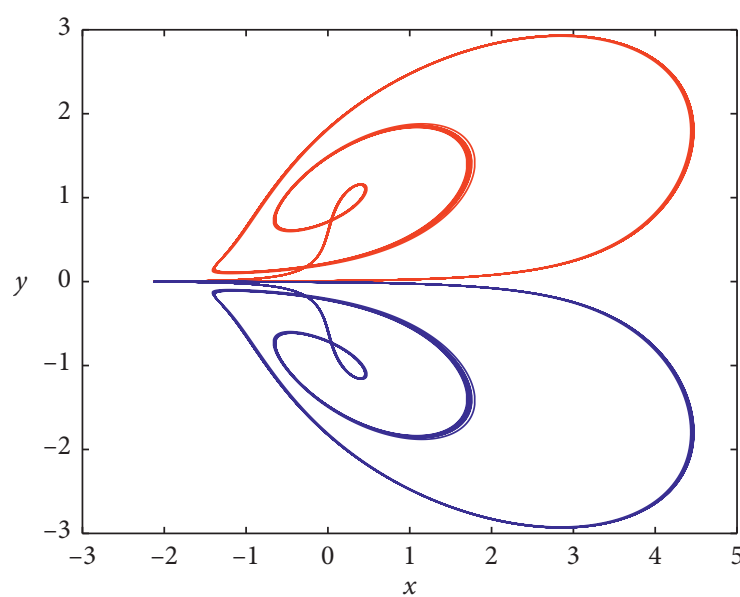

(a)

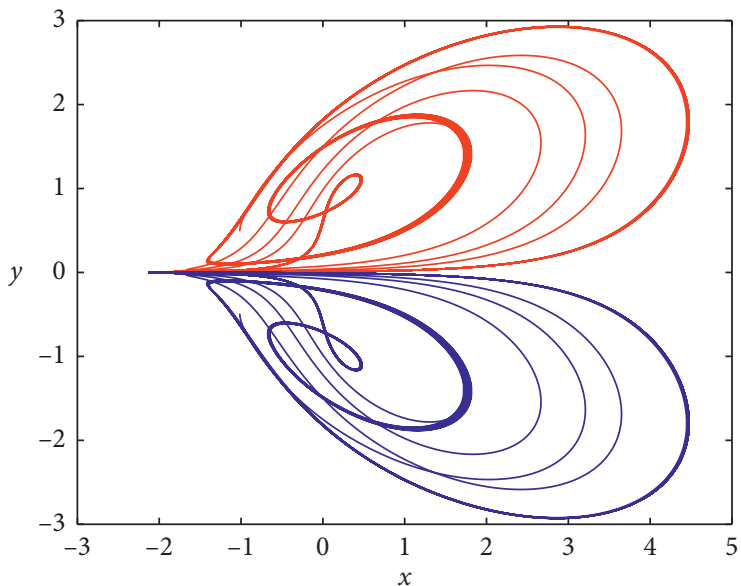

(c)

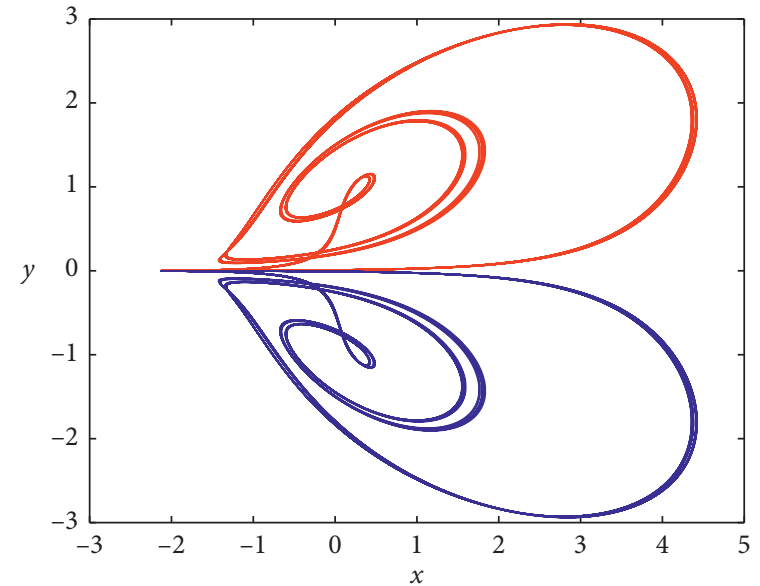

(b)

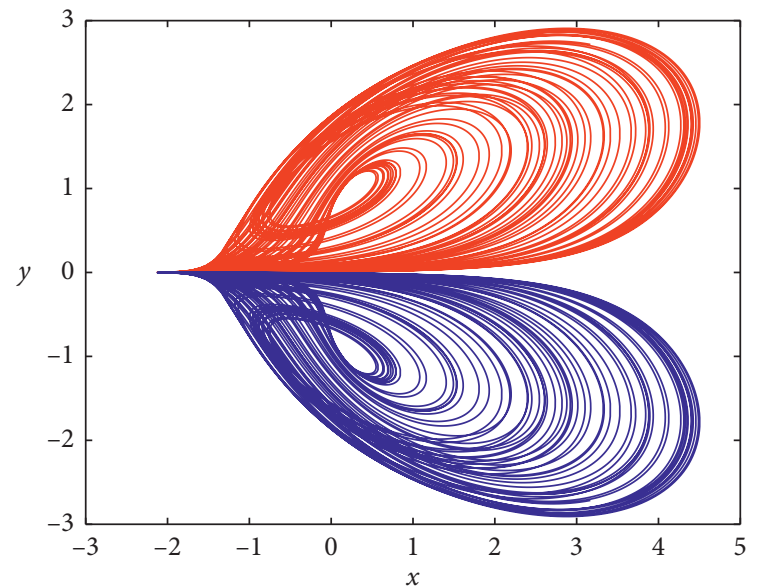

(d)

Figure 10: Coexisting and symmetry attractor of system (2) with $a=5.8$ and $b=7.9$ under the initial condition $\left[1, Y_{0}, 0,5\right]$. (a) Cycle-1 symmetry; (b) cycle-2 symmetry; (c) cycle-4 symmetry; and (d) chaotic symmetry.

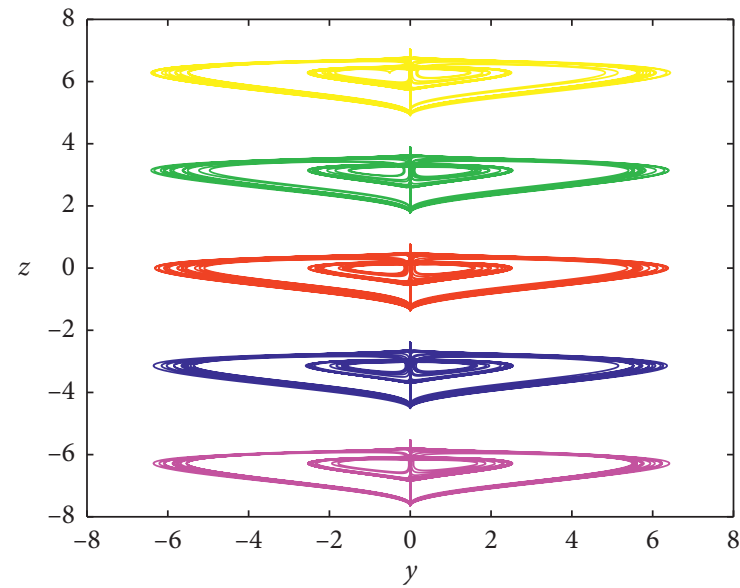

(a)

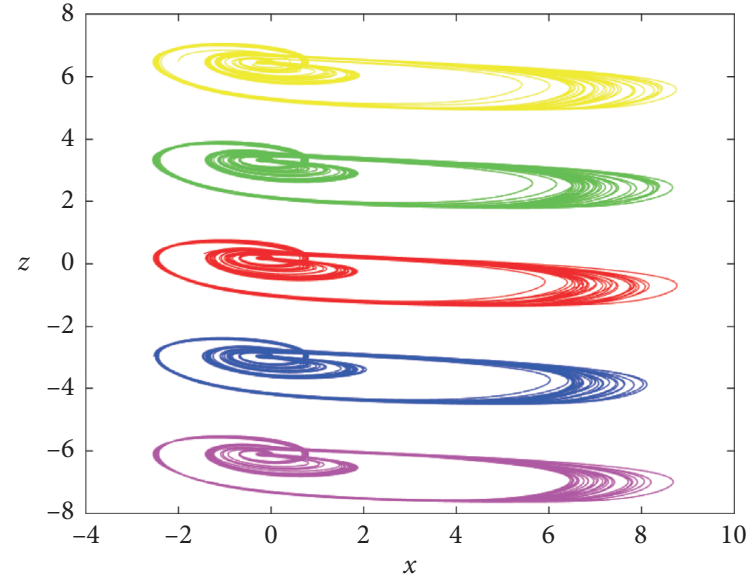

(b)

Figure 11: The self-replicating attractor of system (2), blue (1, 1, -pi, 0); red (1, 1, 0, 5); green (1, 1, pi, 5); yellow (1, 1, $2{ }^{*}$ pi, 0$)$; and kermes $\left(1,1,-2^{*}\right.$ pi, 5). (a) $y$ - $z$ plane; (b) $x$ - $z$ plane. 


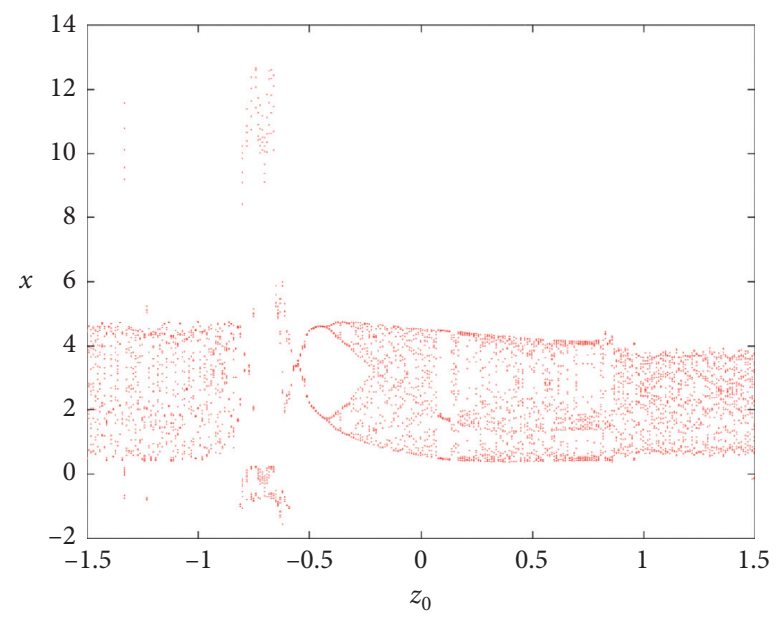

(a)

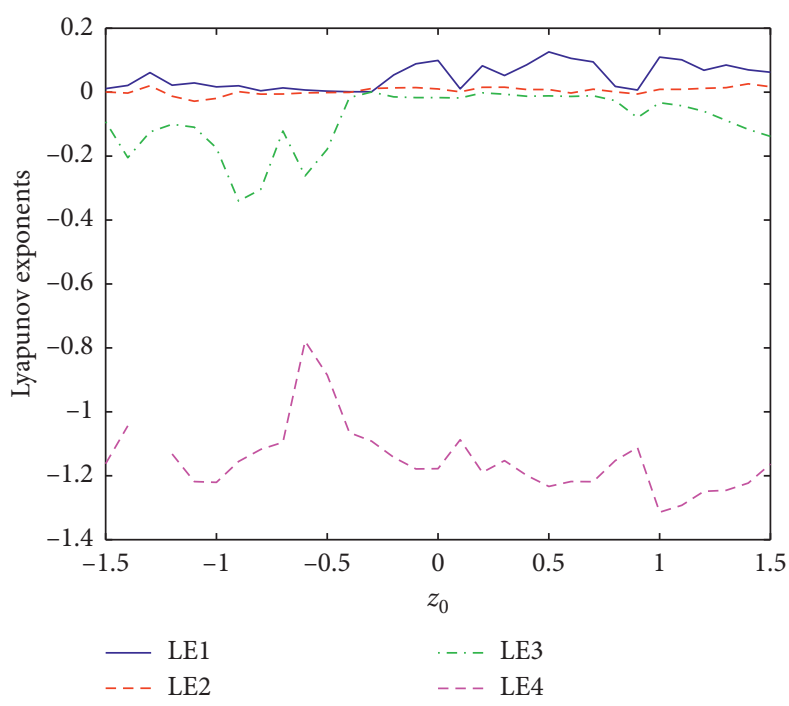

(b)

FiguRE 12: Lyapunov exponents and bifurcation diagram of system (2) with $a=5.8$ and $b=7.9$ under the initial condition [1, $\left.1, z_{0}, 5\right]$. (a) Bifurcation diagram; (b) Lyapunov exponents.

system is in the cycle- 2 state, when the value of $z_{0}$ is 0.12 , the system is in the cycle- 3 state, when the value of $z_{0}$ is -0.32 , the system is in the cycle- 4 state, and when the value of $z_{0}$ is 0.24 , the system is in the quasiperiodic attractor state. When the value of $z_{0}$ is 0.8 , the system is in a chaotic state. Thus, the results of Figure 12 imply that there is coexisting many attractors' behavior in system (3).

\section{Circuit Implementation}

The analog circuit of system (3) is designed as shown in Figure 14 with the following circuit equation:

$$
\left\{\begin{array}{l}
\dot{x}=\frac{1}{R_{1} C_{1}} \tan (z)+\frac{1}{R_{2} C_{1}} y^{2}-\frac{1}{R_{32} C_{1}}, \\
\dot{y}=\frac{1}{R_{16} C_{2}} y \tan (z) \\
\dot{z}=\frac{1}{R_{11} C_{3}} W(u) x-\frac{1}{R_{10} C_{3}} \tan (z), \\
\dot{u}=\frac{1}{R_{20} C_{4}} x .
\end{array}\right.
$$

The circuit consists of four channels to realize the integration, addition, subtraction, and nonlinear operations including absolute value function and quadratic nonlinearity, as shown in Figure 14. The main circuit with the circuit form is plotted in Figure 14(a), where $x, y$, and $z$ represent three state variables of capacitor voltages, respectively, and $\mathrm{RC}$ stands for the time constant of the integrators. The equivalent realization unit circuit of the flux-controlled memristor $W(u)$ is depicted in Figure 14(b), where $u$ is the inner state variable of capacitor voltage in the memristor. The operational amplifier 741 performs the addition and integration, and the analog multiplier AD633 performs the nonlinear product operation. The circuit is powered by $\pm 15 \mathrm{~V}$. When the system parameters $a=5.8 .2$ and $b=7.9$, the corresponding circuit element parameters can be selected as $\mathrm{R} 1=17 \mathrm{~K}, \mathrm{R} 2=\mathrm{R} 32=\mathrm{R} 10=\mathrm{R} 11=\mathrm{R} 20=100 \mathrm{~K} \Omega, \quad \mathrm{R} 3=\mathrm{R} 4=$ $\mathrm{R} 12=\mathrm{R} 13=\mathrm{R} 17=\mathrm{R} 18=\mathrm{R} 22=\mathrm{R} 23=10 \mathrm{~K} \Omega, \mathrm{C} 1=\mathrm{C} 2=\mathrm{C} 3=$ $\mathrm{C} 4=1 \mathrm{nF}$, and $\mathrm{R} 16=12.6 \mathrm{~K} \Omega$. The circuit simulation is shown in Figure 14. The corresponding memristor circuit element parameters can be selected as R $40=\mathrm{R} 36=470 \Omega$, $\mathrm{R} 37=50 \mathrm{~K} \Omega, \mathrm{R} 39=16.6 \mathrm{~K} \Omega$, and $\mathrm{R} 38=1 \mathrm{~K} \Omega$. The circuit simulation diagram and a plot of pinched hysteresis loop of the memristor are shown in Figure 15. The initial voltage of the capacitor is selected as $V_{1}=V_{2}=1 \mathrm{~V}$ and $\mathrm{V}_{2}=-1 \mathrm{~V}$, $V_{3}=0 \mathrm{~V}$, and $V_{4}=5 \mathrm{~V}$, and the circuit simulation is shown in Figure 16(a). It can be seen that the attractors in the $x-y$ phase diagram are symmetrically distributed. The initial voltage of the capacitor is selected as $V_{1}=V_{2}=1 \mathrm{~V}, V_{3}=\pi \mathrm{V}$ and $V_{3}=-\pi \mathrm{V}$, and $V_{4}=5 \mathrm{~V}$, and the circuit simulation is shown in Figure 16(b). It can be seen that the $y$ - $z$ phase diagram shows the growth distribution of attractors. Corresponding to various preset initial conditions given in Figure 8, the PSpice-simulated results are yielded and shown in Figures 17(a) and 17(b). The results in Figures 16 and 17 just verify the complex phenomenon revealed in the Matlab numerical simulations. Additionally, in consideration of different time scales between the mathematical model (3) and the circuit model (9), there exist some differences between the results in Figure 17 and those in Figure 8, but both show the initial-condition-dependent dynamical behavior of period with steady chaos. 


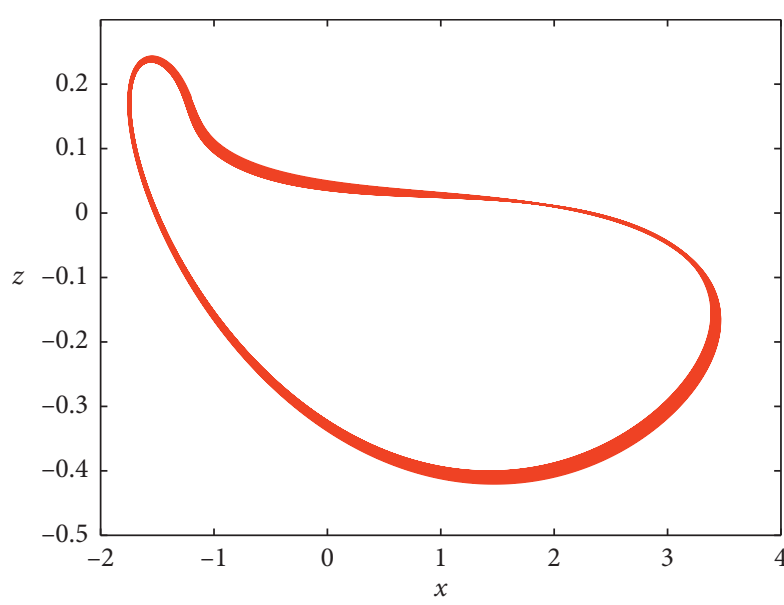

(a)

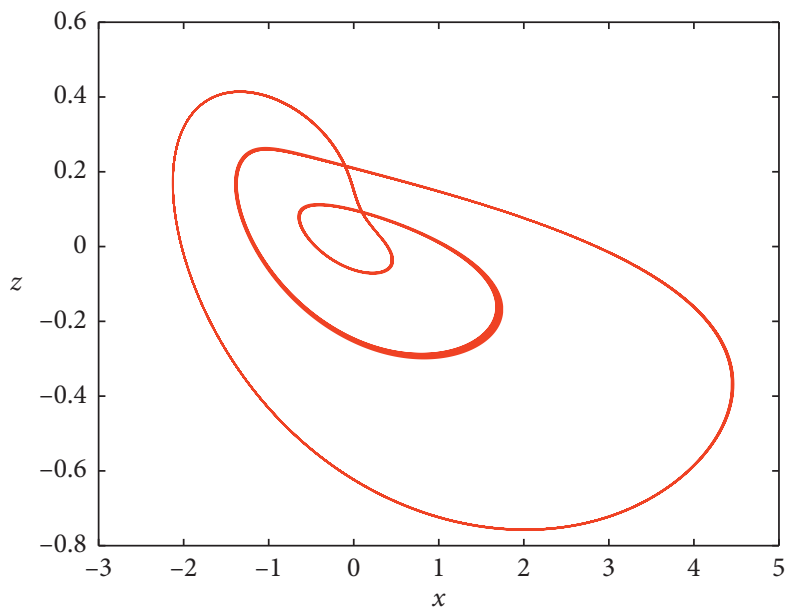

(c)

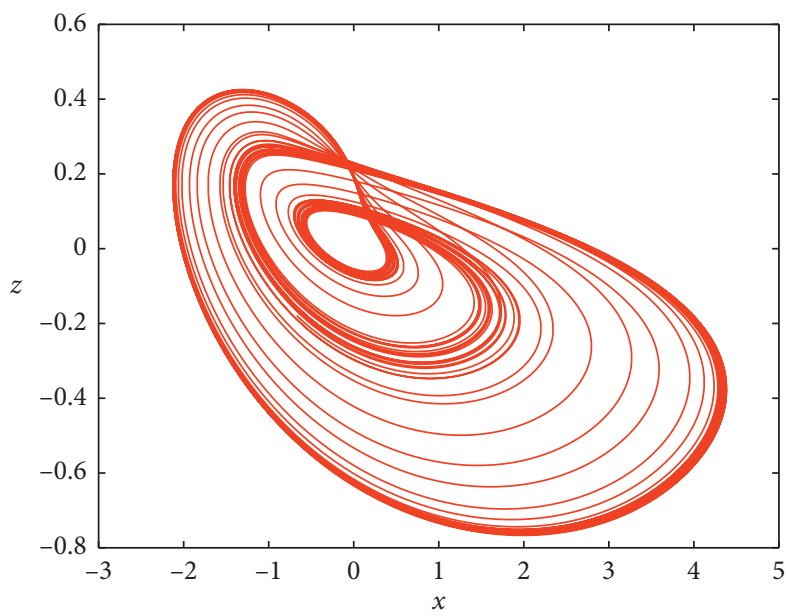

(e)

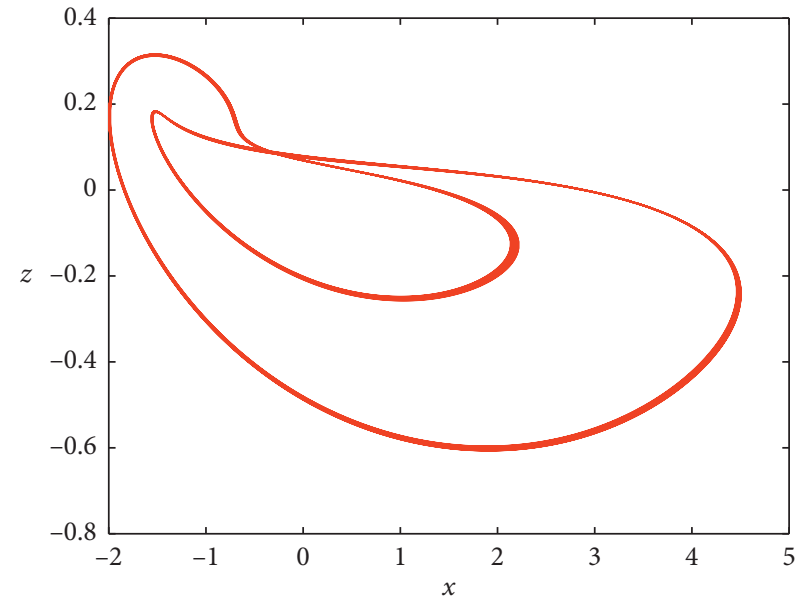

(b)

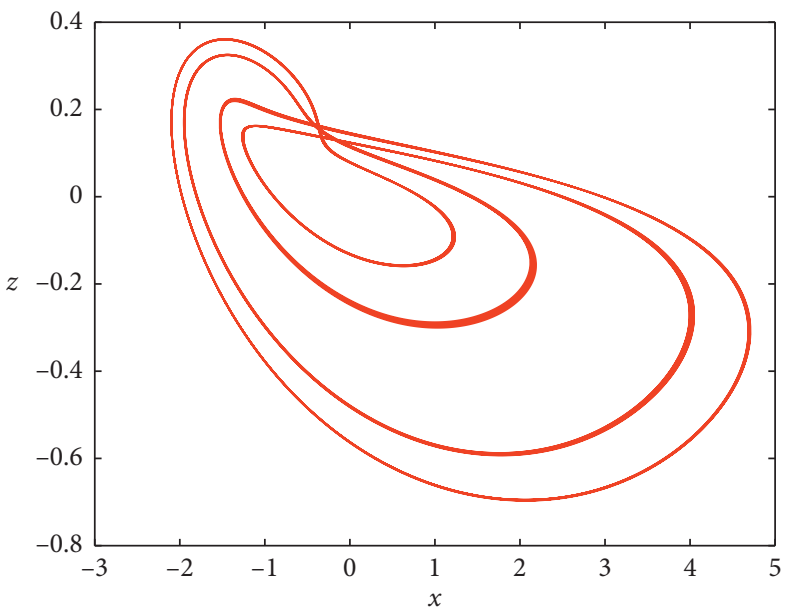

(d)

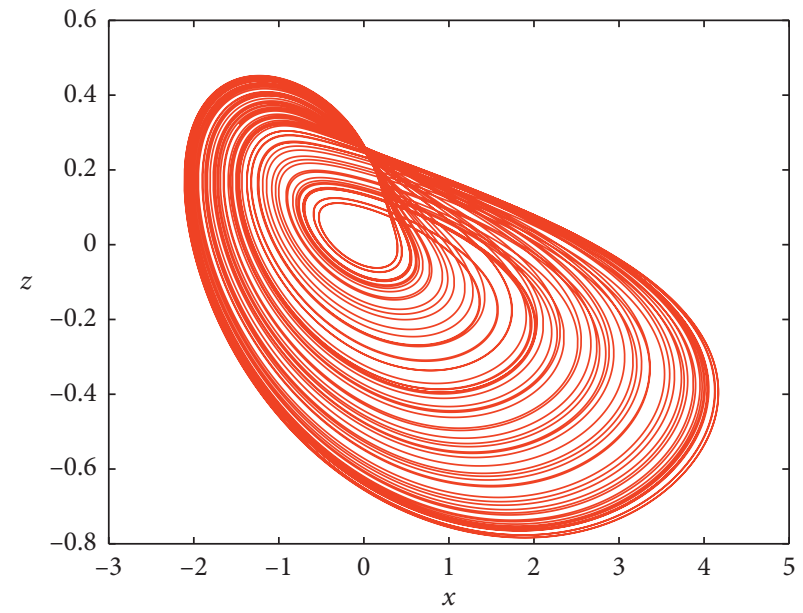

(f)

FIgURe 13: Coexisting attractor of system (2) with $a=5.8$ and $b=7.9$ under the initial condition $\left[1,1, z_{0}, 5\right]$. (a) $z_{0}=-0.55$; (b) $z_{0}=-0.49$; (c) $z_{0}=0.12$; (d) $z_{0}=-0.32$; (e) $z_{0}=0.24$; and (f) $z_{0}=0.8$. 

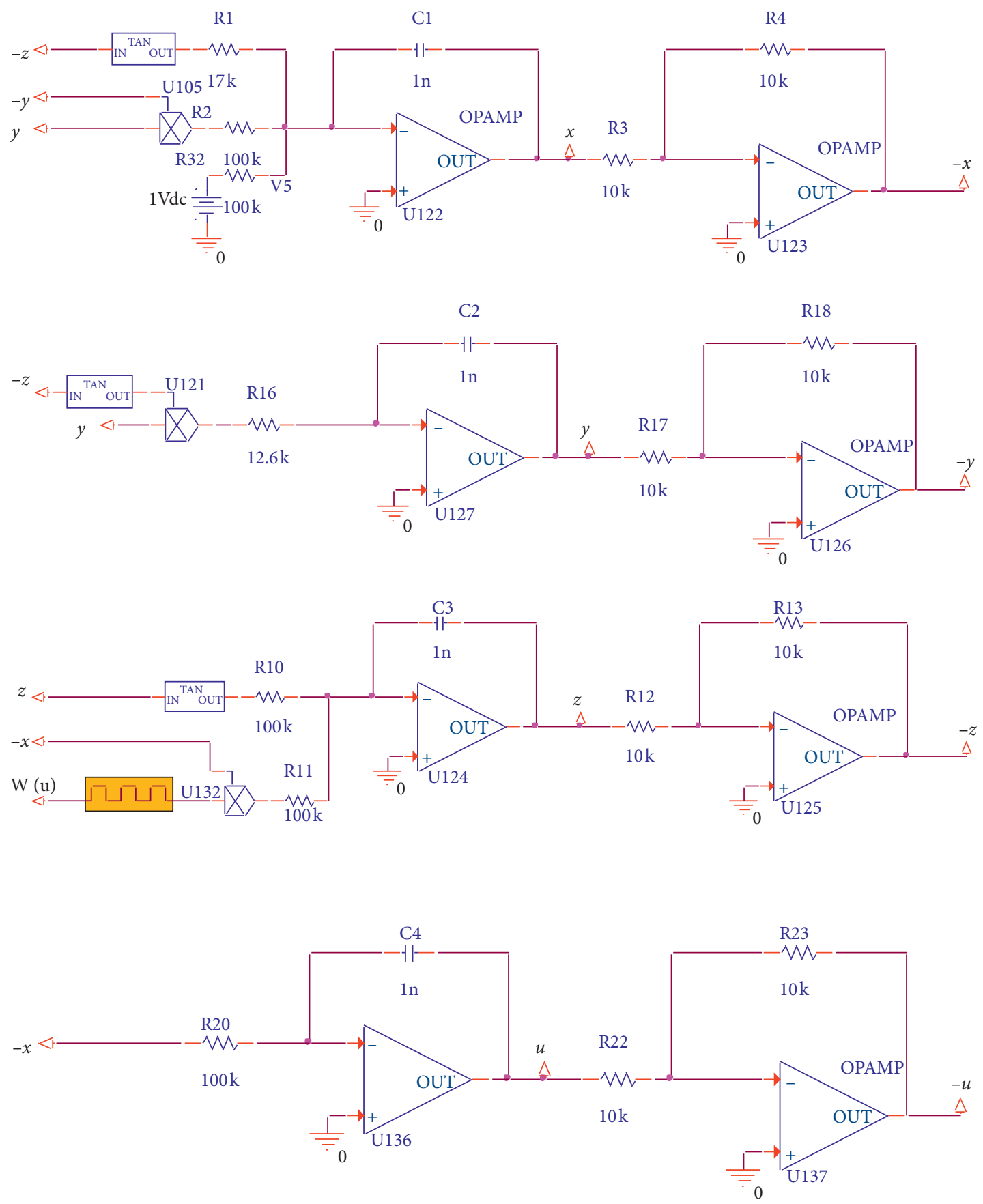

(a)

Figure 14: Continued. 


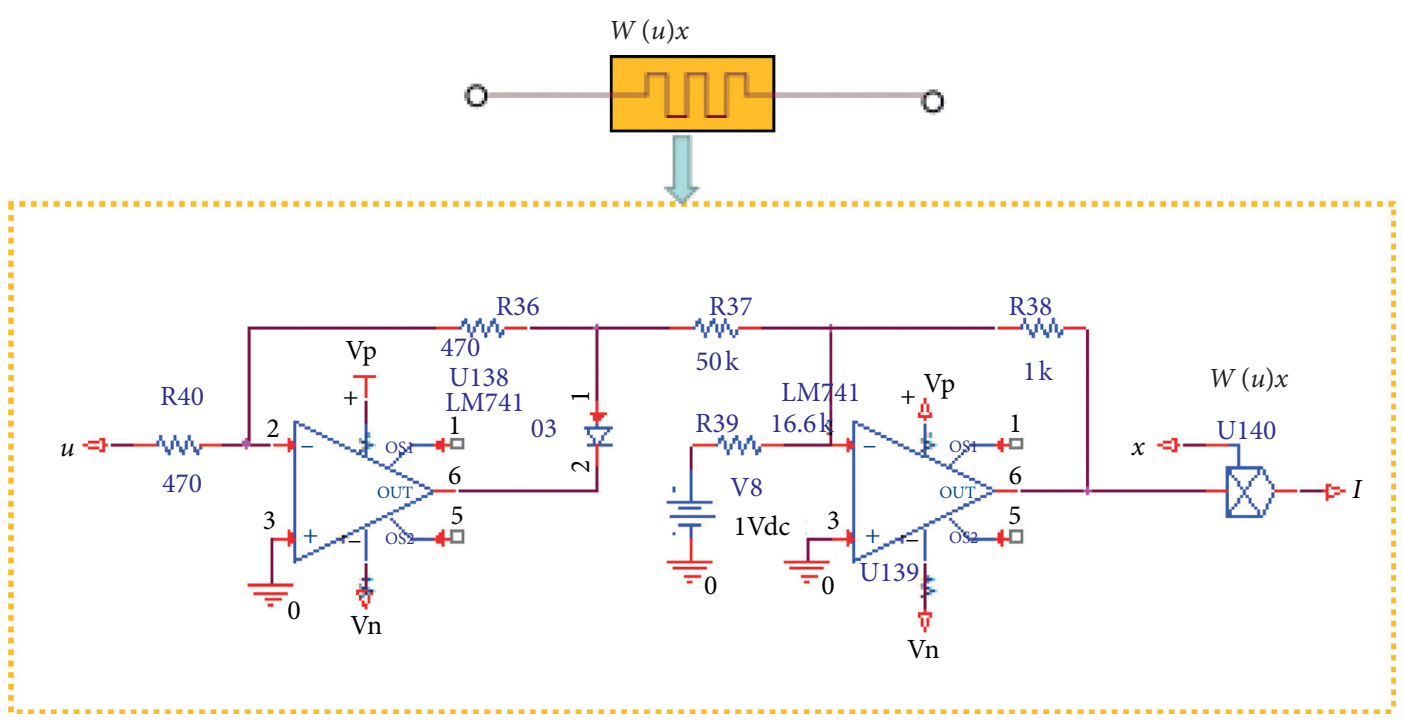

(b)

FIgURE 14: Hardware circuit implementation of memristive system (2): (a) main circuit; (b) memristor equivalent realization unit circuit.

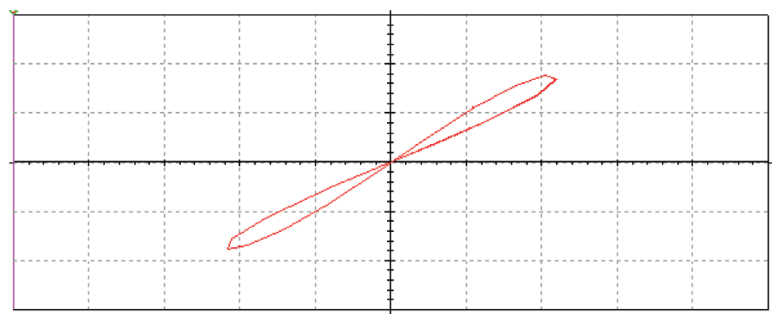

Figure 15: Pinched hysteresis loop of the memristor described by equation (3).

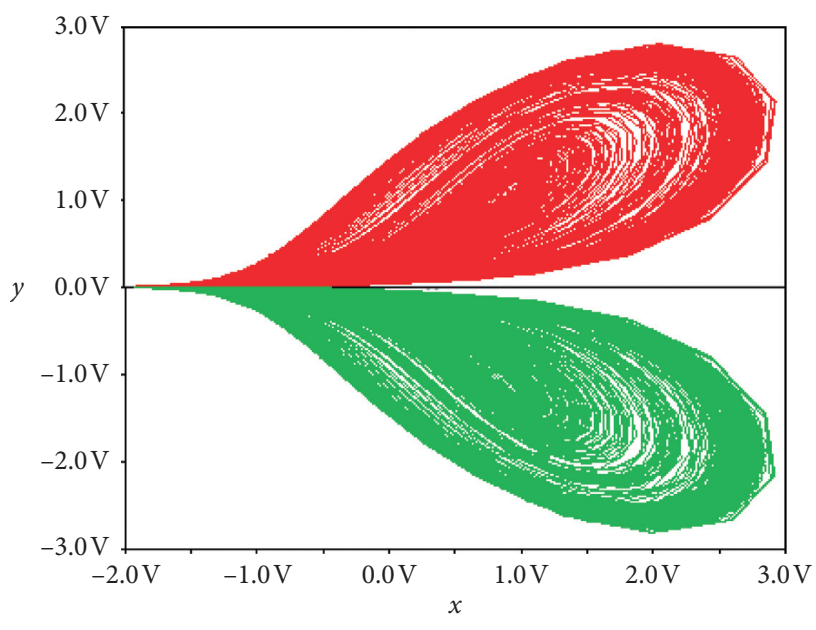

(a)

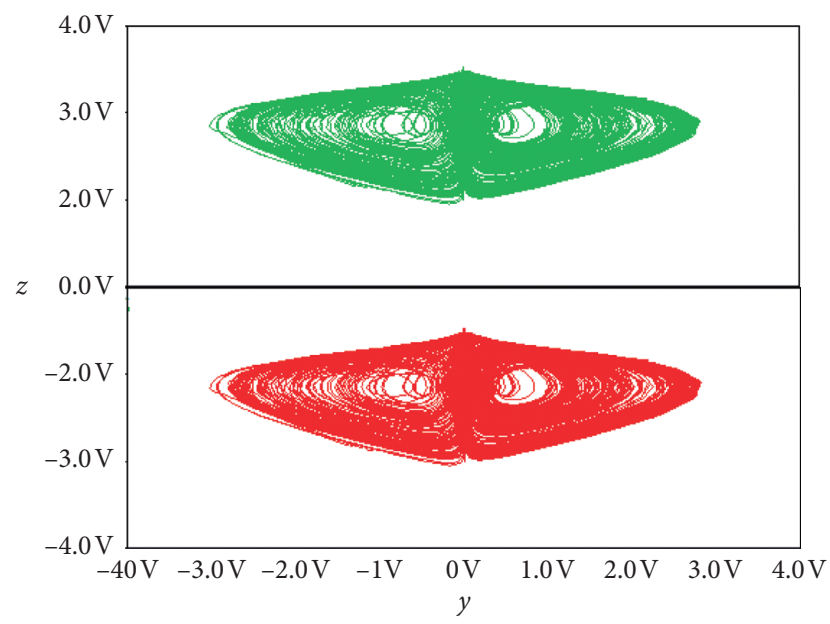

(b)

FiguRE 16: When $a=5.8$ and $b=7.9$, system (3) takes chaotic attractors with different initial values. $[1,1,0,5],[1,-1,0,5],[1,1,-\pi, 0]$, and $[1,1, \pi, 0]$ are red (a), green (a), red (b), and green (b) correspondingly: (a) $x-y$ plane; (b) $y-z$ plane. 


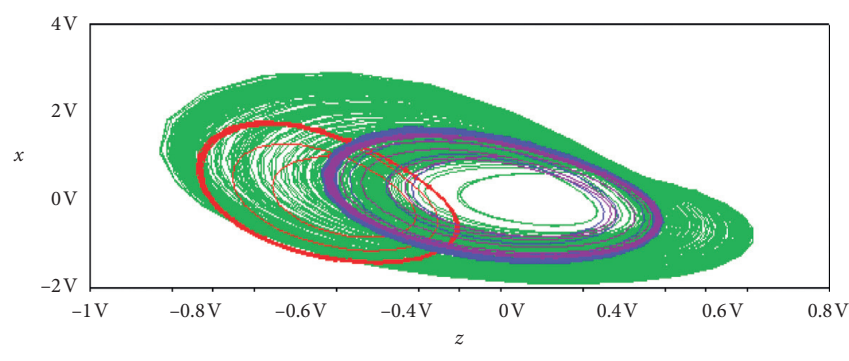

(a)

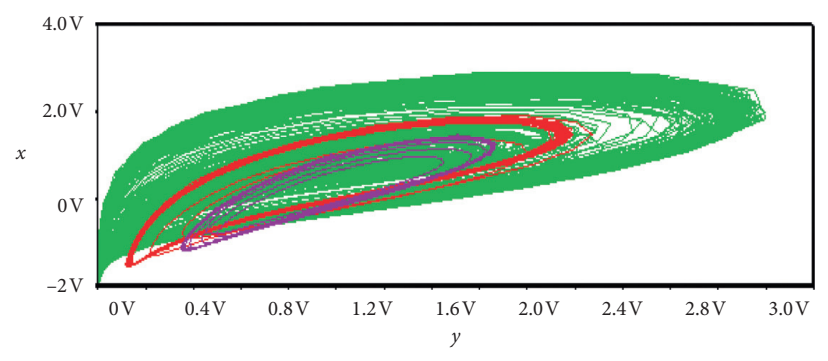

(b)

FIgURE 17: For different initial conditions, phase portraits in the different plane by PSpice: (a) $x-z$ plane; (b) $x-y$ plane.

\section{Conclusions}

A new memristive self-replicating attractor system was obtained by introducing a tangent function and memristor into the classical system. In this case, an infinite line of equilibrium is produced, causing the phenomenon of alternating chaos and period. We analyzed the change of the initial value of each variable in the four-dimensional system, a dynamic behavior in which the transient period and steady state chaos alternately appear depending on the initial value change is newly discovered, and the super multistable state was discussed. The phenomenon of attractor self-replication appears due to the introduction of the tangent function. The results of the new system imply that there is coexisting infinitely many attractors' behavior. The initial-conditiondependent dynamical behaviors of coexisting infinitely many attractors and transient period are finally validated by hardware experiments and PSpice circuit simulations, which could enhance security for possible secure communication.

\section{Data Availability}

The data used to support the findings of this study are available from the corresponding author upon request.

\section{Conflicts of Interest}

The authors declare that there are no conflicts of interest regarding the publication of this paper.

\section{Acknowledgments}

This work was supported in part by the Natural Science Foundation of Shandong Province under Grant ZR2020KA007.

\section{References}

[1] E. N. Lorenz, "Deterministic nonperiodic flow," Journal of the Atmospheric Sciences, vol. 20, no. 2, pp. 130-141, 1963.

[2] X. Wang and G. Chen, "A chaotic system with only one stable equilibrium," Communications in Nonlinear Science and Numerical Simulation, vol. 17, no. 3, pp. 1264-1272, 2012.

[3] Z. G. Shi, S. J. Bi, H. T. Zhang, R. X. Lu, and X. M. Shen, "Improved auxiliary particle filter-based synchronization of chaotic colpitts circuit and its application to secure communication," Wireless Communications and Mobile Computing, vol. 15, no. 10, pp. 1456-1470, 2015.

[4] F. Min, C. Li, L. Zhang, and C. Li, "Initial value-related dynamical analysis of the memristor-based system with reduced dimensions and its chaotic synchronization via adaptive sliding mode control method," Chinese Journal of Physics, vol. 58, pp. 117-131, 2019.

[5] B. C. Bao, Q. Xu, H. Bao, and M. Che, "Extreme multistability in a memristive circuit," Electronics Letters, vol. 53, no. 12, pp. 1008-1010, 2016.

[6] J. Mou, K. Sun, J. Ruan, and S. He, "A nonlinear circuit with two memcapacitors," Nonlinear Dynamics, vol. 86, no. 3, pp. 1735-1744, 2016.

[7] B. C. Bao, H. Bao, N. Wang, M. Chen, and Q. Xu, "Hidden extreme multistability in memristive hyperchaotic system," Chaos, Solitons \& Fractals, vol. 94, pp. 102-111, 2017.

[8] J. Sun, X. Zhao, J. Fang, and Y. Wang, "Autonomous memristor chaotic systems of infinite chaotic attractors and circuitry realization," Nonlinear Dynamics, vol. 94, no. 4, pp. 2879-2887, 2018.

[9] F. Yuan, G. Wang, and X. Wang, "Extreme multistability in a memristor-based multi-scroll hyper-chaotic system," Chaos (Woodbury, NY.), vol. 26, no. 7, p. 073107, 2016.

[10] A. Akgul, C. Arslan, and B. Aricioglu, "Design of an interface for random number generators based on integer and fractional order chaotic systems," Chaos Theory Applications, vol. 1, no. 1, pp. 1-18, 2019.

[11] X. Y. Hu, C. X. Liu, L. Liu, Y. P. Yao, and G. C. Zheng, "Multiscroll hidden attractors and multi-wing hidden attractors in a 5-dimensional memristive system," Chinese Physics B, vol. 11, pp. 124-130, 2017.

[12] H. Wu, Y. Ye, M. Chen, Q. Xu, and B. Bao, "Periodically switched memristor initial boosting behaviors in memristive hypogenetic Jerk system," IEEE Access, vol. 7, pp. 145022145029, 2019.

[13] M. Mantovani, A. D. Armour, W. Belzig, and G. Rastelli, "Dynamical multistability in a quantum-dot laser," Physics B, vol. 99, no. 4, Article ID 045442, 2019.

[14] L. J. Ontañón-García and E. Campos-Cantón, "Widening of the basins of attraction of a multistable switching dynamical system with the location of symmetric equilibria," Nonlinear Analysis: Hybrid Systems, vol. 26, pp. 38-47, 2017.

[15] F. Yuan, Y. Deng, Y. Li, and G. Wang, "The amplitude, frequency and parameter space boosting in a memristormeminductor-based circuit," Nonlinear Dynamics, vol. 96, no. 1, pp. 389-405, 2019.

[16] Y. N. Joglekar and S. J. Wolf, "The elusive memristor: properties of basic electrical circuits," European Journal of Physics, vol. 30, no. 4, pp. 661-675, 2009. 
[17] Z. L. Wang, F. H. Min, and E. R. Wang, "A new hyperchaotic circuit with two memristors and its application in image encryption," AIP Advances, vol. 6, no. 9, pp. 80-83, 2016.

[18] D. W. Yan, L. D. Wang, and S. K. Duan, "Memristor-based multi-scroll chaotic system and its pulse synchronization control," Acta Physica Sinica, vol. 67, no. 11, p. 110502, 2018.

[19] M. E. Sahin, Z. G. Cam Taskiran, H. Guler, and S. E. Hamamci, "Simulation and implementation of memristive chaotic system and its application for communication systems," Sensors and Actuators A: Physical, vol. 290, pp. 107-118, 2019.

[20] L. Wang, T. Dong, and M.-F. Ge, "Finite-time synchronization of memristor chaotic systems and its application in image encryption," Applied Mathematics and Computation, vol. 347, pp. 293-305, 2019.

[21] C. Li, W. Joo-Chen Thio, H. Ho-Ching Iu, and T. Lu, "A memristive chaotic oscillator with increasing amplitude and frequency," IEEE Access, vol. 6, pp. 12945-12950, 2018.

[22] H. Bao, N. Wang, B. Bao, M. Chen, P. Jin, and G. Wang, "Initial condition-dependent dynamics and transient period in memristor-based hypogenetic Jerk system with four line equilibria," Communications in Nonlinear Science and $\mathrm{Nu}$ merical Simulation, vol. 57, pp. 264-275, 2018.

[23] J. Sajad, A. Atefeh, A. J. M. Khalaf et al., "A new hidden chaotic attractor with extreme multi-stability," AEU-International Journal of Electronics and Communications, vol. 89, pp. 131-135, 2018.

[24] W. Wang, Y. C. Zeng, Z. Chen et al., "Coexisting attractors and Hopf bifuracation in floating memristors based chaotic ciruit," Chinese Journal of Computtaional Physics, vol. 34, no. 6, pp. 747-756, 2017.

[25] J. Huang and Y. M. Chen, "Stability and co-dimension one bifuracation analysis of a class of Lorenz chaotic systems with memristor," Advances in Applied Mathematics, vol. 8, no. 4, pp. 858-867, 2019.

[26] Y. M. Chen, "Research on complex dynamics of four dimensional hyper-chaotic systems based on Lorenz-type systems," Ph.D thesis, South China University of Technology, Guangzhou, China, 2014.

[27] G. Peng and F. Min, "Multistability analysis, circuit implementations and application in image encryption of a novel memristive chaotic circuit," Nonlinear Dynamics, vol. 90, no. 3, pp. 1607-1625, 2017.

[28] J. Liu, S. Liu, and C. Yuan, "Adaptive complex modified projective synchronization of complex chaotic (hyperchaotic) systems with uncertain complex parameters," Nonlinear Dynamics, vol. 79, no. 2, pp. 1035-1047, 2015.

[29] Q. Xu, Y. Lin, B. Bao, and M. Chen, "Multiple attractors in a non-ideal active voltage-controlled memristor based Chua's circuit," Chaos, Solitons \& Fractals, vol. 83, pp. 186-200, 2016.

[30] L Zhou and C. Wang, "Generating four-wing hyperchaotic attractor and two-wing, three-wing, and four-wing chaotic attractors in 4D memristive system," International Journal of Bifurcation and Chaos, vol. 27, no. 2, p. 1750027, 2017.

[31] C. Li and J. C. Sprott, "Variable-boostable chaotic flows," Optik, vol. 127, no. 22, pp. 10389-10398, 2016.

[32] X. Yi, R. Guo, and Y. Qi, "Stabilization of chaotic systems with both uncertainty and disturbance by the UDE-based control method," IEEE Access, vol. 8, no. 1, pp. 62471-62477, 2020.

[33] L. Liu, B. Li, and R. Guo, "Consensus control for networked manipulators with switched parameters and topologies," IEEE Access, vol. 9, pp. 9209-9217, 2021.
[34] T. Hou, Y. Y. Liu, and F. Q. Deng, "Stability for discrete-time uncertain systems with infinite Markov jump and time-delay," Science China-Information Sciences, vol. 64, pp. 1-11, 2021. 